\title{
A Systematic Review with Meta-Analysis of the Effect of Resistance Training on Whole-Body Muscle Growth in Healthy Adult Males
}

\author{
Pedro J. Benito ${ }^{1, *\left(\mathbb{C}, \text { Rocío Cupeiro }^{1}\left(\mathbb{D}, \text { Domingo J. Ramos-Campo }^{2}(\mathbb{D} \text {, Pedro E. Alcaraz }\right.\right.}{ }^{2,3}$ and \\ Jacobo Á. Rubio-Arias ${ }^{1,2}$ (1) \\ 1 LFE Research Group, Department of Health and Human Performance, Faculty of Physical Activity and Sport \\ Science-INEF, Universidad Politécnica de Madrid, 28040 Madrid, Spain; rocio.cupeiro@upm.es (R.C.); \\ ja.rubio@upm.es (J.Á.R.-A.) \\ 2 Department of Physical Activity and Sports Sciences, Faculty of Sports, UCAM, Catholic University \\ San Antonio, 30107 Murcia, Spain; djramos@ucam.edu (D.J.R.-C.); palcaraz@ucam.edu (P.E.A.) \\ 3 UCAM Research Centre for High Performance Sport, Catholic University San Antonio, 30107 Murcia, Spain \\ * Correspondence: pedroj.benito@upm.es; Tel.: +34-910-677-989
}

Received: 30 December 2019; Accepted: 12 February 2020; Published: 17 February 2020

check for updates

\begin{abstract}
We performed a systematic review and meta-analysis to study all published clinical trial interventions, determined the magnitude of whole-body hypertrophy in humans (healthy males) and observed the individual responsibility of each variable in muscle growth after resistance training (RT). Searches were conducted in PubMed, Web of Science and the Cochrane Library from database inception until 10 May 2018 for original articles assessing the effects of RT on muscle size after interventions of more than 2 weeks of duration. Specifically, we obtain the variables fat-free mass (FMM), lean muscle mass (LMM) and skeletal muscle mass (SMM). The effects on outcomes were expressed as mean differences (MD) and a random-effects meta-analysis and meta-regressions determined covariates (age, weight, height, durations in weeks ... ) to explore the moderate effect related to the participants and characteristics of training. One hundred and eleven studies (158 groups, 1927 participants) reported on the effects of RT for muscle mass. RT significantly increased muscle mass (FFM+LMM+SMM; $\left.\Delta 1.53 \mathrm{~kg} ; 95 \% \mathrm{CI}[1.30,1.76], p<0.001 ; \mathrm{I}^{2}=0 \%, p=1.00\right)$. Considering the overall effects of the meta-regression, and taking into account the participants' characteristics, none of the studied covariates explained any effect on changes in muscle mass. Regarding the training characteristics, the only significant variable that explained the variance of the hypertrophy was the sets per workout, showing a significant negative interaction (MD; estimate: 1.85, 95\% CI [1.45, 2.25], $p<0.001$; moderator: $-0.0395 \%$ CI $[-0.05,-0.001] p=0.04)$. In conclusion, RT has a significant effect on the improvement of hypertrophy $(\sim 1.5 \mathrm{~kg})$. The excessive sets per workout affects negatively the muscle mass gain.
\end{abstract}

Keywords: hypertrophy; fat-free mass; skeletal muscle mass; lean muscle mass; resistance training

\section{Introduction}

Achieving a proper muscle mass is a key factor for sports performance as well as for attaining a good body image. Furthermore, muscle is increasingly being recognized as a key tissue for the maintenance of an adequate health status, not only regarding movement and posture but also as a regulator of inter-organ crosstalk for energy and protein metabolism throughout the body [1]. Therefore, improving lean body mass and preventing muscle loss should be crucial in promoting overall health and in achieving the targeted performance level [1,2]. 
In this context, being able to predict changes in body composition would be very useful for training and conditioning and for health professionals, both in the non-clinical and the clinical fields, helping them to set appropriate goals as well as to correctly assess the progression during interventions. However, despite its importance, several limitations arise regarding muscle hypertrophy, defined as an increase in the size of muscle tissue [3]. On the one hand, no consensus exists for quantifying this increase in muscle tissue, since different methods are employed to measure it (fat-free mass, lean muscle mass or skeletal muscle mass). On the other hand, another limitation for trainers and practitioners is that with current knowledge and research it is difficult to predict how much muscle mass can be gained.

Several studies have already designed estimation equations to predict weight evolution throughout a weight loss programme [4,5], and although an intrinsic uncertainty and error margin of those estimations are always present, the same method of evaluation for muscle gain is necessary as we have already pointed out. In this sense, two pioneer works have paved the way to the prediction of muscle hypertrophy. Firstly, the meta-analysis by Morton et al. [6] has shown how supplementation can influence muscle hypertrophy, explicitly reporting the weight of this variable (i.e., supplementation) over the levels of muscle gain. Secondly, based on the models proposed by Hall et al. [5], Torres et al. created a mathematical model to predict muscle hypertrophy in humans. Nevertheless, the work by Morton et al. [6] focuses on the effect of protein intake on muscle gain, without addressing the effect of training without protein supplementation; while the model created by Torres et al. is a theoretical model focused on fat loss and lean mass gain in obese people, not determining the effect of the different training parameters. In fact, the authors declare that further models may be developed and redefined to include parameters they had not addressed [7]. Therefore, it seems that a really integrative model, taking into account all the variables that influence muscle hypertrophy, is currently lacking.

In this respect, muscle hypertrophy in humans is influenced by numerous variables, from those inherent to exercise such as mechanical tension, metabolic stress, muscle damage, training volume and intensity [8,9]; to those being specific to the individual (age, sex, previous training status, etc.) [10], or those related to energy balance and protein metabolism [6,7]. Consequently, any study aiming to analyse and estimate muscle hypertrophy in humans must precisely address all confounding factors that may completely alter the final results.

As mentioned above, only two studies have attempted to estimate the changes in muscle mass after a training intervention [6,7]. However, none of them handle the effect of these confounding factors that we have mentioned. Therefore, the effect of an isolated training programme on muscle hypertrophy, the variation in its design, age or resistance training (RT) experience, are currently unknown. Thus, the aims of the present study were: (i) to quantify the impact of strength training on human whole-body muscle hypertrophy, and (ii) to analyse training factors which could moderate the human muscle growth in men.

\section{Materials and Methods}

\subsection{Study Design}

The methodological process was based on the recommendations indicated by the PRISMA declaration [11]. The eligibility criteria were established by the authors. For the meta-analysis, research that studied resistance training was considered. The study was preregistered in the International Prospective Register of Systematic Review (PROSPERO) with the following registered number: CRD42018106274.

\subsection{Data Sources and Search Profile}

A comprehensive literature search was performed using PubMed-Medline, Web of Science and the Cochrane Library from database inception through to 10 May 2018. The database searches were performed independently by two authors (P.J.B. and D.J.R.-C.) and the results obtained were the same. 
The following combination terms was used: "muscle hypertrophy" or "muscle thickness" or "muscle gain" or "fat free mass" or "muscular size" or "muscle size" or "skeletal muscle mass" or "appendicular skeletal muscle mass" or "muscle mass" or "body fat free". The Boolean operator "AND" was used to combine these descriptors with: "resistance training" or "strength training" or "weight training" or "power training" or "weightlifting" or "full body" or "circuit" or "neuromuscular training" or "body weight training". Also, the Boolean operator "NOT" was combined with the terms "sarcopenia" or "atroph" or "Review". The flow diagram of the search process is shown in Figure 1.
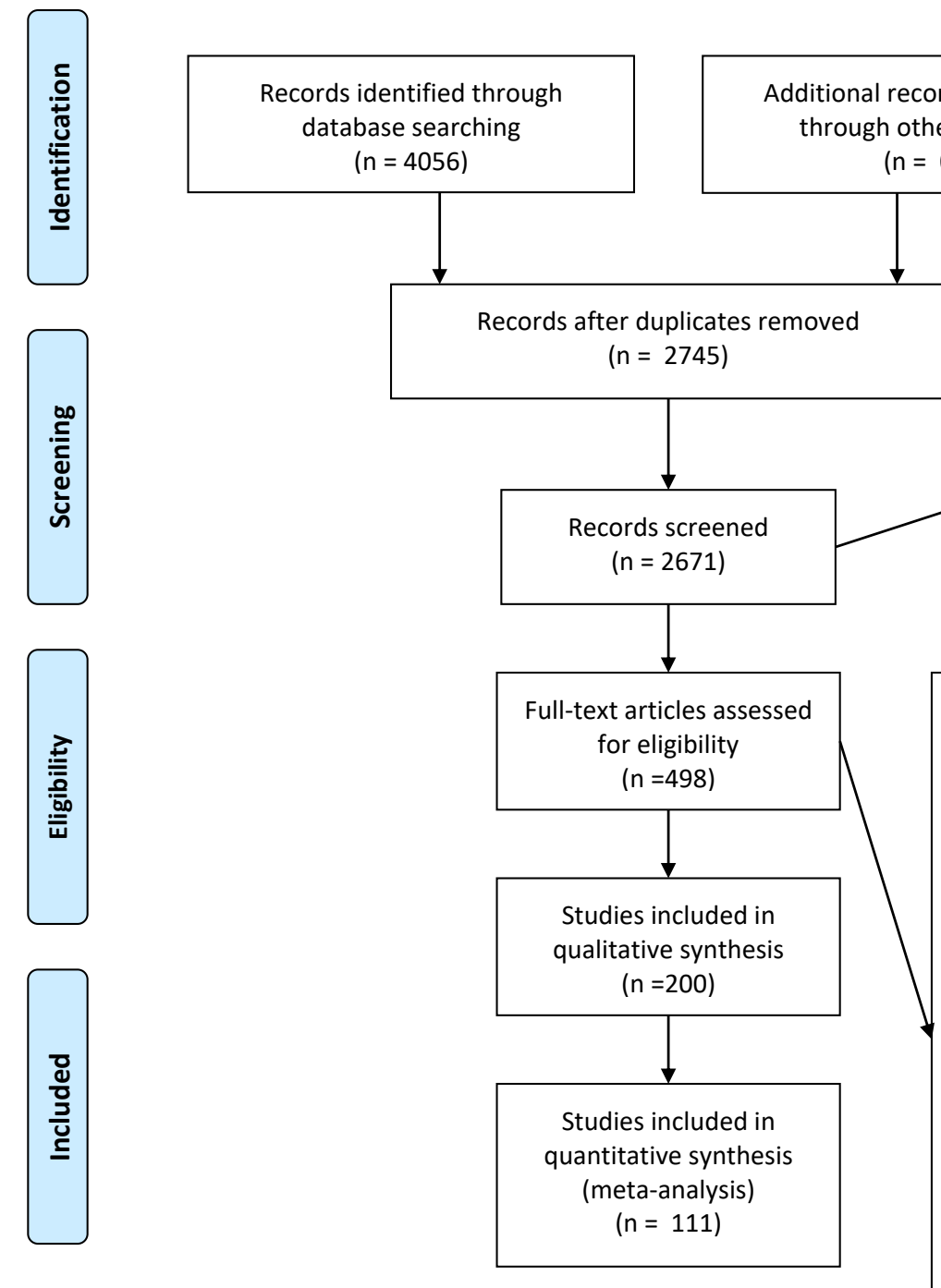

Full-text articles excluded, with reasons $(n=290)$.

Not intervention desing or Review. $(\mathrm{n}=$ 12)

No healthy or specific patologies. $(n=11)$ No specific measurement of muscle mass in whole body $(n=61)$ Age not between 18 and 40 years old ( $n=139$ )

No gravitional weight training i.e. (elastic, pool RT, vibrations, concurrent training) ( $n=16)$

Negative energy balance $(n=3)$

Meeting abstract $(n=11)$ Language $(n=1)$

Men and women in the same group ( $\mathrm{n}=$ 31)

Women $(n=1)$

Duplicate data of studies $(n=4)$

Missing data ( $n$, mean or SD) $(n=19)$

Programe duration longer that 1 year $(n=1)$

Figure 1. Prisma flow diagram for the included studies.

\subsection{Selection Criteria}

The specific inclusion criteria were: (1) studies examining the effect of RT from for at least 2 weeks to one year on lean body mass, skeletal muscle mass or fat-free mass; (2) participants were men; (3) studies published in English, and; (4) studies provide information of outcomes both at baseline and follow-up. Research studies were excluded if they: (1) used a sample population with pathologies or not between 18 and 40 years of age; (2) used men and women in the same group of training; (3) performed RT with a negative energy balance; (4) were a review or did not have an 
intervention design; (5) were not an original investigation published in full; (6) did not specify the tests to be evaluated or did not use a specific measurement of muscle mass in whole body; (7) applied gravitational weight training (i.e., elastic bands, pool resistance training, concurrent training, body vibrations ... ); (8) did not provide or specify numerical data; and, (9) they examined acute effects of interventions.

\subsection{Study Selection and Data Extraction}

Retrieved articles were reviewed independently by two authors (P.J.B. and D.J.R.-C.), to choose potentially relevant articles; all disagreements on inclusion/exclusion were discussed and resolved by consensus. References of potentially relevant articles were also searched to find additional studies, and authors of selected studies were contacted for non-reported information. Two authors (P.J.B. and D.J.R.-C.) independently extracted data from the included studies. The following information was extracted: authors of the paper, study design, number of participants included in each group, training experience and training status, age, weight and height. Regarding the characteristics of the RT programmes, the information extracted included: the type of exercise (i.e., full body, circuit training, traditional split routine ... ), duration of training (weeks), training frequency (sessions/week), number of exercise performed each day, relative load lifted, sets and repetitions performed, rest between sets and between repetitions, total number of sessions, nutritional supplementation, drugs use and the outcomes measured (e.g., lean body mass, skeletal muscle mass or fat-free mass).

\subsection{Outcomes}

The output variables of the articles were defined as follows. Fat-free mass (FMM) was calculated as "all that is not fat", subtracting fat weight from body weight, or when the measurements were obtained by dual X-ray absorptiometry was calculated as lean tissue plus bone mineral content [12]. Lean muscle mass (LMM), lean mass, lean body mass, bone-free lean body mass or mineral-free lean mass was calculated as the fat-free mass minus the bone mineral content (DXA) or as fat-free mass minus the estimated weight [13] of the live bone by the equation of Heymsfield et al. [14]. Skeletal muscle mass (SMM) or skeletal muscle was defined as lean muscle and was calculated by anthropometric equations, by proprietary algorithms when using bioimpedance or by estimates based on dual X-ray absorptiometry data [15].

\subsection{Evaluation of the Methodology of the Studies Selected}

The methodological quality of the selected studies was assessed with the quality assessment tool for before-after (pre-post) studies with no control group tool (National Heart Lung and Blood Institute, 2014), which analyses the following items: (1) study question; (2) eligibility criteria and study population; (3) study participants representative of clinical populations of interest; (4) all eligible participants enrolled; (5) sample size; (6) intervention clearly described; (7) outcome measures clearly described, valid, and reliable; (8) blinding of outcome assessors; (9) follow-up rate; (10) statistical analysis; (11) multiple outcome measures; and (12) group-level interventions and individual-level outcome efforts. For each study, each item was described as having either a low risk of bias, an unclear risk of bias or a high risk of bias. Risk of bias was assessed independently by two authors (P.J.B. and D.J.R.-C.) using the previously described risk of bias tool.

\subsection{Data Synthesis and Statistical Analysis}

The meta-analysis and the statistical analysis were conducted using the Review Manager software (RevMan 5.2; Cochrane Collaboration, Oxford, UK). A random effects meta-analysis was conducted to determine the summary effect of RT on lean body mass, skeletal muscle mass or fat-free mass. The effects of training on these outcomes were expressed as mean differences (MD) and their 95\% confidence intervals (CI). The heterogeneity between the studies was evaluated through the $\mathrm{I}^{2}$ statistic, and between-study variance using the tau-square (Tau2) [16]. The $\mathrm{I}^{2}$ values of $30-60 \%$ represented 
a moderate level of heterogeneity. A $p$ value $<0.1$ suggests the presence of substantial statistical heterogeneity. The publication bias was evaluated through an asymmetry test as estimated from a funnel plot (Figure 2). In addition, the Egger's test was used to assess publication bias. A p-value of less than 0.05 was considered to be statistically significant.

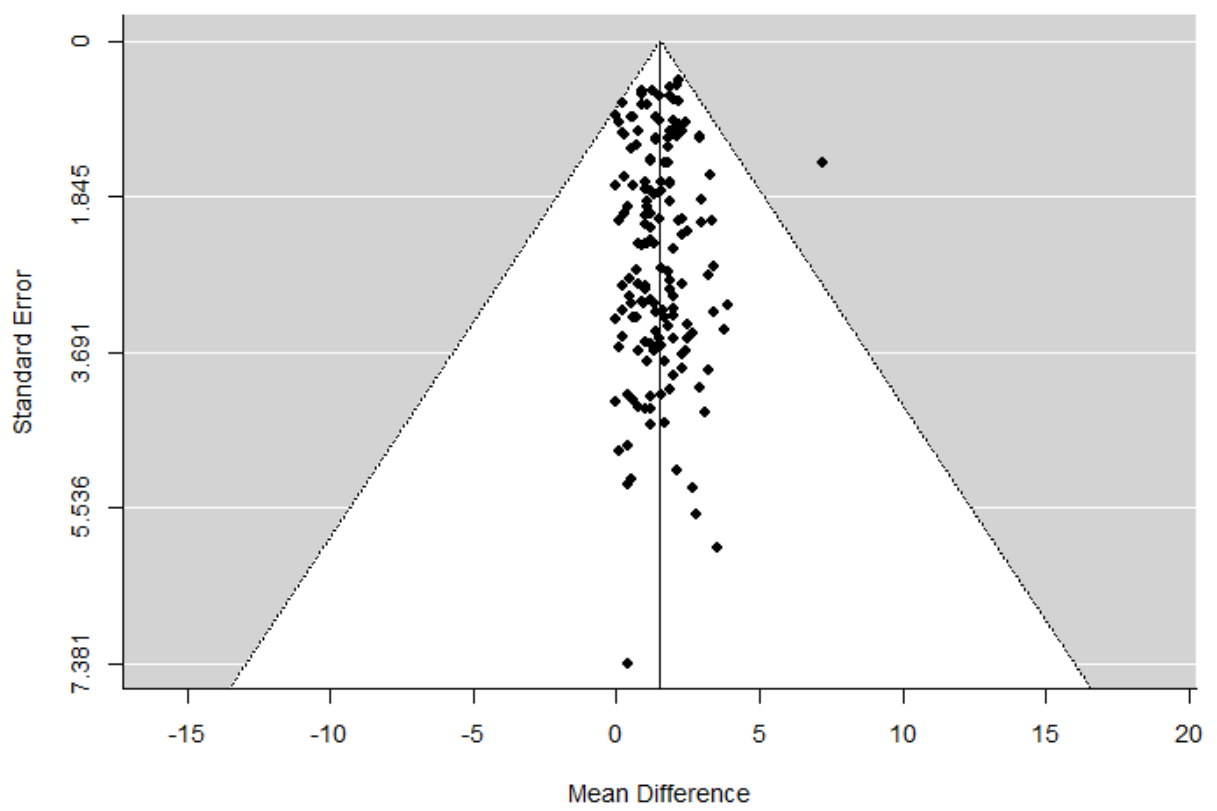

Figure 2. Test for funnel plot asymmetry of the change of muscle mass (all variables measured: FFM, LMM, SMM) after resistance training.

Effects of Moderator Variables: Meta-Regression and Sub-Analysis

To explore the moderate effect related to the participants and characteristics of training, meta-regression and meta-analysis were performed. The continuous covariates were meta-regressed individually and together in a random-effects meta-regression model using Jamovi project (Package for R). The following prognostic factors were considered: average of age, weight, height, study durations (weeks), sessions, days per week, number of exercises per workout, rest between exercise, number of sets per workout, range repetitions, training duration ( $\mathrm{min}$ ) and average intensity (\%1RM) and for training status the studies were coded according to the following structure: sedentary/untrained $=0$; physically active but no experience in RT $=1$; RT up to 1 year/intermediate $=2$; RT experience up to 2 years/intermediate $=3$; RT experience up to 3 years/intermediate $=4$; RT experience of 4 years or more/advanced $=5$. For the meta-regression, we used a residual restricted maximum likelihood to measure between-study variance $(\tau 2)$. Factors found to be significant at $p=0.05$ level were included in multivariate meta-regression models. In addition, the training status variable was considered to be a categorical variable and in order to do so the participants categorized as untrained were those described as untrained, without experience in RT or less than one year of experience with loads, and the participants with RT experience of more than one year were categorized as trained. Finally, subgroup analyses were used for the effects of/to find the effects of categorical variables (training status: untrained vs trained).

\section{Results}

\subsection{General Characteristics of Studies}

The initial search, which was based on the effect of resistance training on muscle mass, identified 4056 articles from the databases and no articles from other sources. After removing the duplicates, 2671 abstracts were screened, 2173 were excluded and 498 were screened as full texts. Finally, 111 studies 
(see Supplementary Table S1) were determined to fulfil the inclusion criteria and thus selected for the meta-analysis (Figure 1). Publications ranged from 1973 to 2018. The instruments used to carry out the measurements were anthropometry (n groups $=19)$, ultrasound (n groups $=1)$, Bod Pod (n groups = 3), bioelectrical impedance analysis (BIA) (n groups $=14$ ), dual-energy X-ray absorptiometry (DXA) (n groups $=35$ ), hydrodensitometry, hydrostatic weighing and underwater weighing (n groups $=10$ ) FFM; anthropometry (n groups $=4$ ), BIA (n groups $=1$ ), DXA (n groups $=48$ ), underwater weighing ( $\mathrm{n}$ groups $=12$ ) for LMM and anthropometry (n groups $=8$ ) and DXA ( $\mathrm{n}$ groups $=3$ ) for SMM. The main characteristics and properties of the included studies are summarized in Supplementary Table S1.

\subsubsection{The Participants' Characteristics}

The initial search, which was based on the effect of resistance training on muscle mass, identified 111 articles (158 groups) and 1927 participants were measured ( $23.5 \pm 3.31$ years; $79.4 \pm 6.42 \mathrm{~kg}$ and $177 \pm 9.19 \mathrm{~cm}$ ). Sixty-one studies (82 groups) analysed fat-free mass, 52 studies (65 groups) evaluated the lean muscle mass and seven studies (11 groups) examined the muscle mass to analyze the effects of a resistance training programme on muscle mass. Some of these studies measured more than one variable and the same study may be included in FFM, LMM or SMM (see Table 1). There were a total of 951 participants in fat-free mass (23.8 \pm 3.40 years; $79.5 \pm 6.36 \mathrm{~kg}$ and $178 \pm 3.64 \mathrm{~cm}), 810$ participants in lean body mass $(23.2 \pm 3.47$ years; $80.4 \pm 6.41 \mathrm{~kg}$ and $176 \pm 1.40 \mathrm{~cm})$ and 155 participants in muscle mass $(23.0 \pm 1.24$ years; $73.5 \pm 3.5 \mathrm{~kg} 179 \pm 3.0 \mathrm{~cm})$. The participants who carried out the intervention had a state of training between untrained to bodybuilder professionals; the frequency of each state was sedentary/untrained ( $\mathrm{n}$ groups: $\mathrm{FFM}=13 ; \mathrm{LMM}=3 ; \mathrm{SMM}=0$ ); physically active without experience (n groups: $\mathrm{FFM}=26 ; \mathrm{LMM}=13 ; \mathrm{SMM}=3$ ); with experience in resistance training up to one year (n groups: $\mathrm{FFM}=8 ; \mathrm{LMM}=14 ; \mathrm{SMM}=5$ ); with experience $1-2$ years (n groups: $\mathrm{FFM}=8$; $\mathrm{LMM}=5$; $\mathrm{SMM}=0$ ); with experience $2-4$ years (n groups: $\mathrm{FFM}=8 ; \mathrm{LMM}=6 ; \mathrm{SMM}=0$ ); and with experience 4 years or more (n groups: $\mathrm{FFM}=7 ; \mathrm{LMM}=0 ; \mathrm{SMM}=0$ ).

Table 1. Main differences between pre-post study in the main body composition variable considered. The numerical suffix after the references indicate the different subgroup in the same study. See supplemental material for more information.

\begin{tabular}{ccccccccc}
\hline Study/Group/Year/Reference & \multicolumn{2}{c}{ Post-Training } & \multicolumn{2}{c}{ Pre-Training } & Weight & Mean Difference \\
\hline & Mean & SD & $\mathbf{n}$ & Mean & SD & $\mathbf{n}$ & & Random, 95\% IC \\
\hline Fat Free Mass (FFM) & & & & & & & & \\
\hline Abe et al., 2000 [17] & 62.7 & 5.3 & 17 & 61.1 & 5.1 & 17 & $0.90 \%$ & $1.60[-1.90,5.10]$ \\
\hline Ahtiainen et al., 2011 [18] & 68.0 & 4.1 & 7 & 65.0 & 4.0 & 7 & $0.60 \%$ & $3.00[-1.24,7.24]$ \\
\hline Álvarez, et al., 2012_1 [19] & 60.0 & 6.6 & 5 & 56.9 & 7.3 & 5 & $0.10 \%$ & $3.10[-5.53,11.73]$ \\
\hline Álvarez, et al., 2012_2 [19] & 59.2 & 6.8 & 5 & 58.2 & 7.0 & 5 & $0.20 \%$ & $1.00[-7.55,9.55]$ \\
\hline Alvehus et al., 2014 [20] & 65.5 & 7.0 & 17 & 63.2 & 6.4 & 17 & $0.50 \%$ & $2.30[-2.21,6.81]$ \\
\hline Arazi et al., 2015_1 [21] & 63.6 & 6.5 & 10 & 61.7 & 6.2 & 10 & $0.40 \%$ & $1.90[-3.67,7.47]$ \\
\hline Arciero et al., 2001_1 [22] & 64.7 & 3.6 & 10 & 63.0 & 2.8 & 10 & $1.40 \%$ & $1.70[-1.13,4.53]$ \\
\hline Arciero et al., 2001_2 [22] & 62.5 & 6.5 & 10 & 62.3 & 6.5 & 10 & $0.30 \%$ & $0.20[-5.50,5.90]$ \\
\hline Bang et al., 2017_1 [23] & 70.9 & 10.2 & 8 & 70.5 & 10.8 & 8 & $0.10 \%$ & $0.40[-9.89,10.69]$ \\
\hline Bartolomei et al., 2016_1 [24] & 73.8 & 7.5 & 10 & 72.0 & 7.6 & 10 & $0.30 \%$ & $1.80[-4.82,8.42]$ \\
\hline Bartolomei et al., 2016_2 [24] & 70.6 & 9.4 & 8 & 69.4 & 8.8 & 8 & $0.10 \%$ & $1.21[-7.71,10.13]$ \\
\hline Bartolomei et al., 2018_1 [12] & 75.4 & 7.5 & 9 & 73.7 & 8.6 & 9 & $0.20 \%$ & $1.70[-5.76,9.16]$ \\
\hline Bartolomei et al., 2018_2 [12] & 73.5 & 7.7 & 11 & 72.8 & 7.7 & 11 & $0.30 \%$ & $0.70[-5.74,7.14]$ \\
\hline Bhasin et al., 1996_1 [25] & 74.1 & 2.2 & 9 & 72.1 & 2.3 & 9 & $2.50 \%$ & $2.00[-0.08,4.08]$ \\
\hline
\end{tabular}


Table 1. Cont.

\begin{tabular}{|c|c|c|c|c|c|c|c|c|}
\hline \multirow[t]{2}{*}{ Study/Group/Year/Reference } & \multicolumn{3}{|c|}{ Post-Training } & \multicolumn{3}{|c|}{ Pre-Training } & \multirow[t]{2}{*}{ Weight } & \multirow{2}{*}{$\begin{array}{l}\text { Mean Difference } \\
\text { Random, } 95 \% \text { IC }\end{array}$} \\
\hline & Mean & SD & $\mathrm{n}$ & Mean & SD & $\mathrm{n}$ & & \\
\hline Buresh et al., 2009_1 [26] & 74.7 & 3.7 & 6 & 72.4 & 3.6 & 6 & $0.60 \%$ & $2.30[-1.83,6.43]$ \\
\hline Buresh et al., 2009_2 [26] & 65.6 & 7.9 & 6 & 64.4 & 6.7 & 6 & $0.20 \%$ & $1.20[-7.09,9.49]$ \\
\hline Chromiak et al., 2004_1 [27] & 71.4 & 9.5 & 18 & 68.0 & 9.8 & 18 & $0.30 \%$ & $3.40[-2.91,9.71]$ \\
\hline Chromiak et al., 2004_2 [27] & 68.3 & 9.9 & 15 & 66.8 & 9.8 & 15 & $0.20 \%$ & $1.50[-5.55,8.55]$ \\
\hline Colquhoun et al., 2017_1 [28] & 72.2 & 5.4 & 11 & 71.4 & 5.9 & 11 & $0.50 \%$ & $0.80[-3.93,5.53]$ \\
\hline Crewther et al., 2013 [29] & 77.7 & 5.2 & 12 & 76.7 & 5.5 & 12 & $0.60 \%$ & $1.00[-3.28,5.28]$ \\
\hline Deruisseau et al., 2004 [30] & 64.5 & 8.6 & 13 & 63.1 & 9.0 & 13 & $0.20 \%$ & $1.40[-5.37,8.17]$ \\
\hline Fu et al., 2017_1 [31] & 63.4 & 9.8 & 9 & 62.2 & 8.7 & 9 & $0.10 \%$ & $1.19[-7.37,9.75]$ \\
\hline Fu et al., 2017_2 [31] & 62.8 & 7.7 & 9 & 61.5 & 7.8 & 9 & $0.20 \%$ & $1.35[-5.85,8.55]$ \\
\hline Gallagher et al., 2000_1 [32] & 65.3 & 2.2 & 14 & 65.3 & 2.5 & 14 & $3.60 \%$ & $0.00[-1.74,1.74]$ \\
\hline Gallagher et al., 2000_2 [32] & 66.3 & 1.6 & 12 & 64.4 & 1.6 & 12 & $6.70 \%$ & $1.90[0.62,3.18]$ \\
\hline Garthe et al., 2013_1 [33] & 65.0 & 8.7 & 21 & 63.4 & 8.7 & 21 & $0.40 \%$ & $1.60[-3.67,6.87]$ \\
\hline Garthe et al., 2013_2 [33] & 65.6 & 6.2 & 18 & 64.6 & 6.3 & 18 & $0.70 \%$ & $1.00[-3.07,5.07]$ \\
\hline Ghahramanloo et al., 2009 [34] & 57.1 & 6.1 & 9 & 55.2 & 6.4 & 9 & $0.30 \%$ & $1.90[-3.88,7.68]$ \\
\hline Hong et al., 2014_1 [35] & 64.7 & 7.6 & 8 & 62.0 & 6.3 & 8 & $0.20 \%$ & $2.66[-4.15,9.47]$ \\
\hline Hong et al., 2014_2 [35] & 63.8 & 8.1 & 10 & 60.0 & 7.2 & 10 & $0.20 \%$ & $3.80[-2.93,10.53]$ \\
\hline Hu et al., 2009 [36] & 66.5 & 6.7 & 48 & 65.3 & 7.0 & 48 & $1.50 \%$ & $1.20[-1.54,3.94]$ \\
\hline Huso et al., 2002_2 [37] & 65.4 & 1.8 & 10 & 63.2 & 1.4 & 10 & $5.50 \%$ & $2.20[0.79,3.61]$ \\
\hline Jang et al., 2017_1 [38] & 60.3 & 6.2 & 8 & 58.9 & 6.7 & 8 & $0.30 \%$ & $1.40[-4.93,7.73]$ \\
\hline Joy et al., 2014_1 [39] & 60.7 & 4.7 & 14 & 59.5 & 4.7 & 14 & $0.90 \%$ & $1.20[-2.29,4.69]$ \\
\hline Kim et al., 2014 [40] & 52.7 & 4.1 & 9 & 51.5 & 4.6 & 9 & $0.70 \%$ & $1.20[-2.83,5.23]$ \\
\hline Kon et al., 2014 [41] & 55.8 & 7.9 & 7 & 53.8 & 6.9 & 7 & $0.20 \%$ & $2.00[-5.78,9.78]$ \\
\hline Kramer et al., 2009_2 [42] & 61.6 & 6.8 & 9 & 57.7 & 6.5 & 9 & $0.30 \%$ & $3.90[-2.23,10.03]$ \\
\hline Kreipke et al., 2015_1 [43] & 62.9 & 4.9 & 13 & 61.8 & 4.8 & 13 & $0.80 \%$ & $1.10[-2.63,4.83]$ \\
\hline Lemmer et al., 2001 [44] & 64.9 & 7.1 & 10 & 62.9 & 7.1 & 10 & $0.30 \%$ & $2.00[-4.23,8.23]$ \\
\hline Li et al., 2014 [45] & 71.7 & 2.8 & 13 & 70.6 & 8.2 & 13 & $0.50 \%$ & $1.04[-3.69,5.77]$ \\
\hline Lockwood et al., 2017_1 [46] & 61.7 & 8.5 & 15 & 60.4 & 8.5 & 15 & $0.30 \%$ & $1.30[-4.79,7.39]$ \\
\hline Lukaski et al., 1996_1 [47] & 67.3 & 2.2 & 12 & 65.9 & 2.2 & 12 & $3.50 \%$ & $1.40[-0.36,3.16]$ \\
\hline Lukaski et al., 1996_2 [47] & 65.9 & 1.5 & 12 & 64.0 & 1.2 & 12 & $9.30 \%$ & $1.90[0.81,2.99]$ \\
\hline Lukaski et al., 1996_3 [47] & 66.1 & 2.6 & 12 & 64.2 & 2.6 & 12 & $2.50 \%$ & $1.90[-0.18,3.98]$ \\
\hline Mazzetti et al., 2000_1 [48] & 69.6 & 2.6 & 10 & 68.2 & 2.6 & 10 & $2.10 \%$ & $1.38[-0.89,3.65]$ \\
\hline Mazzetti et al., 2000_2 [48] & 68.4 & 1.5 & 8 & 68.2 & 1.5 & 8 & $5.20 \%$ & $0.25[-1.20,1.70]$ \\
\hline McCarthy et al., 1995 [49] & 68.1 & 2.3 & 10 & 65.9 & 2.1 & 10 & $3.00 \%$ & $2.20[0.27,4.13]$ \\
\hline Nacleiro et al., 2017_1 [50] & 64.2 & 7.3 & 8 & 64.2 & 7.3 & 8 & $0.20 \%$ & $0.08[-7.05,7.21]$ \\
\hline Noonan et al., 1998_1 [51] & 88.3 & 10.4 & 13 & 85.1 & 9.5 & 13 & $0.20 \%$ & $3.20[-4.46,10.86]$ \\
\hline Oliver et al., 2013_1 [52] & 71.9 & 9.8 & 11 & 70.0 & 9.6 & 11 & $0.20 \%$ & $1.90[-6.21,10.01]$ \\
\hline Oliver et al., 2013_2 [52] & 72.3 & 9.9 & 11 & 71.9 & 9.8 & 11 & $0.20 \%$ & $0.40[-7.83,8.63]$ \\
\hline Ormsbee et al., 2012_1 [53] & 64.7 & 5.9 & 11 & 63.5 & 5.2 & 11 & $0.50 \%$ & $1.20[-3.45,5.85]$ \\
\hline Ormsbee et al., 2013_1 [54] & 68.2 & 6.0 & 11 & 66.9 & 5.3 & 11 & $0.50 \%$ & $1.30[-3.43,6.03]$ \\
\hline Piirainen et al., 2008_1 [55] & 65.9 & 6.6 & 6 & 65.3 & 8.1 & 6 & $0.20 \%$ & $0.60[-7.76,8.96]$ \\
\hline Piirainen et al., 2008_2 [55] & 64.3 & 9.4 & 6 & 63.8 & 8.6 & 6 & $0.10 \%$ & $0.50[-9.69,10.69]$ \\
\hline Pérez-Gómez et al., 2013 [56] & 59.7 & 7.7 & 8 & 58.6 & 7.5 & 8 & $0.20 \%$ & $1.09[-6.35,8.53]$ \\
\hline Radaelli et al., 2015_1 [57] & 67.7 & 6.5 & 12 & 67.2 & 8.3 & 12 & $0.30 \%$ & $0.46[-5.49,6.41]$ \\
\hline
\end{tabular}


Table 1. Cont

\begin{tabular}{|c|c|c|c|c|c|c|c|c|}
\hline \multirow[t]{2}{*}{ Study/Group/Year/Reference } & \multicolumn{3}{|c|}{ Post-Training } & \multicolumn{3}{|c|}{ Pre-Training } & \multirow[t]{2}{*}{ Weight } & \multirow{2}{*}{$\begin{array}{l}\text { Mean Difference } \\
\text { Random, } 95 \% \text { IC }\end{array}$} \\
\hline & Mean & SD & $\mathrm{n}$ & Mean & SD & $\mathbf{n}$ & & \\
\hline Radaelli et al., 2015_2 [57] & 66.0 & 5.2 & 13 & 63.0 & 4.4 & 13 & $0.80 \%$ & $2.98[-0.71,6.67]$ \\
\hline Radaelli et al., 2015_3 [57] & 74.7 & 5.0 & 13 & 71.4 & 5.9 & 13 & $0.60 \%$ & $3.32[-0.89,7.53]$ \\
\hline Ribeiro et al., 2015_1 [58] & 75.0 & 6.4 & 5 & 72.1 & 6.6 & 5 & $0.20 \%$ & $2.90[-5.16,10.96]$ \\
\hline Ribeiro et al., 2015_2 [58] & 76.5 & 9.9 & 5 & 73.0 & 9.1 & 5 & $0.10 \%$ & $3.50[-8.29,15.29]$ \\
\hline Roberts et al., 2007_2 [59] & 64.9 & 8.0 & 16 & 63.9 & 8.4 & 16 & $0.30 \%$ & $1.00[-4.68,6.68]$ \\
\hline Rogerson et al., 2007_2 [60] & 78.0 & 8.4 & 11 & 76.8 & 8.4 & 11 & $0.20 \%$ & $1.20[-5.82,8.22]$ \\
\hline Schumann et al., 2014_1 [61] & 62.9 & 2.6 & 16 & 60.9 & 2.8 & 16 & $3.10 \%$ & $2.00[0.13,3.87]$ \\
\hline Schumann et al., 2014_2 [61] & 60.7 & 3.6 & 18 & 59.3 & 3.5 & 18 & $2.00 \%$ & $1.40[-0.92,3.72]$ \\
\hline Shelmadine et al., 2009_2 [62] & 55.8 & 6.8 & 9 & 54.9 & 6.4 & 9 & $0.30 \%$ & $0.95[-5.16,7.06]$ \\
\hline Snijders et al., 2015_1 [63] & 64.8 & 6.1 & 19 & 62.9 & 5.7 & 19 & $0.80 \%$ & $1.90[-1.85,5.65]$ \\
\hline Spence et al., 2011 [64] & 65.5 & 9.7 & 13 & 63.1 & 9.0 & 13 & $0.20 \%$ & $2.40[-4.79,9.59]$ \\
\hline Spillane et al., 2009_3 [65] & 56.3 & 10.2 & 10 & 54.6 & 10.1 & 10 & $0.10 \%$ & $1.70[-7.18,10.58]$ \\
\hline Spillane et al., 2011_1 [66] & 57.0 & 9.9 & 19 & 56.4 & 10.3 & 19 & $0.30 \%$ & $0.59[-5.83,7.01]$ \\
\hline Terzis et al., 2008 [67] & 65.4 & 9.6 & 8 & 65.0 & 9.6 & 8 & $0.10 \%$ & $0.40[-9.01,9.81]$ \\
\hline Terzis et al., 2008b [68] & 62.6 & 6.6 & 11 & 62.2 & 6.6 & 11 & $0.40 \%$ & $0.44[-5.08,5.96]$ \\
\hline Thomson et al., 2009_2 [69] & 61.8 & 5.4 & 17 & 61.4 & 6.1 & 17 & $0.70 \%$ & $0.40[-3.47,4.27]$ \\
\hline Tomljanović et al., 2011 [70] & 69.2 & 10.4 & 23 & 68.3 & 10.6 & 23 & $0.30 \%$ & $0.92[-5.15,6.99]$ \\
\hline Wilborn, et al., 2010_2 [71] & 69.6 & 8.1 & 13 & 67.9 & 8.3 & 13 & $0.30 \%$ & $1.64[-4.64,7.92]$ \\
\hline Willoughby et al., 2007_2 [72] & 68.8 & 10.3 & 10 & 63.2 & 9.4 & 10 & $0.10 \%$ & $5.62[-3.02,14.26]$ \\
\hline Willoughby et al., 2014_1 [73] & 63.9 & 11.9 & 9 & 61.2 & 10.6 & 9 & $0.10 \%$ & $2.70[-7.69,13.09]$ \\
\hline Wilson et al., 2013_1 [74] & 70.5 & 7.6 & 10 & 68.5 & 8.2 & 10 & $0.20 \%$ & $2.00[-4.93,8.93]$ \\
\hline Wilson et al., 2014_1 [75] & 69.2 & 1.1 & 9 & 67.1 & 1.1 & 9 & $10.60 \%$ & $2.10[1.08,3.12]$ \\
\hline Zamani et al., 2017_1 [76] & 56.2 & 1.3 & 10 & 54.9 & 1.3 & 10 & $8.30 \%$ & $1.26[0.11,2.41]$ \\
\hline Zamani et al., 2017_2 [76] & 54.6 & 1.6 & 10 & 53.7 & 1.2 & 10 & $6.90 \%$ & $0.91[-0.35,2.17]$ \\
\hline Zamani et al., 2017_3 [76] & 50.5 & 5.9 & 10 & 49.8 & 6.3 & 10 & $0.40 \%$ & $0.72[-4.63,6.07]$ \\
\hline Total $(95 \% \mathrm{CI})$ & & & 951 & & & 951 & $100.00 \%$ & $1.56[1.23,1.89]$ \\
\hline \multicolumn{5}{|c|}{ Heterogeneity: $\mathrm{Tau}^{2}=0.00 ; \mathrm{Chi}^{2}=18.14, \mathrm{df}=81(p=1.00) ; \mathrm{I}^{2}=0 \%$} & \multicolumn{4}{|c|}{ Test for overall effect: $Z=9.22(p<0.00001)$} \\
\hline \multicolumn{9}{|l|}{ Lean Muscle Mass (LMM) } \\
\hline Alcaraz et al., 2011_1 [77] & 56.4 & 5.3 & 11 & 55.2 & 5.9 & 11 & $0.5 \%$ & $1.20[-3.49,5.89]$ \\
\hline Alcaraz et al., 2011_2 [77] & 60.3 & 5.2 & 15 & 58.8 & 4.6 & 15 & $1.0 \%$ & $1.50[-2.01,5.01]$ \\
\hline Alvehus et al., 2014 [20] & 59.0 & 6.5 & 17 & 56.8 & 6.0 & 17 & $0.7 \%$ & $2.20[-2.00,6.40]$ \\
\hline Andre et al., 2016_1 [78] & 60.5 & 9.1 & 10 & 58.9 & 9.7 & 10 & $0.2 \%$ & $1.60[-6.64,9.84]$ \\
\hline Ara et al., 2006 [79] & 56.5 & 4.2 & 12 & 55.9 & 4.2 & 12 & $1.1 \%$ & $0.60[-2.76,3.96]$ \\
\hline Bemben et al., 2001_2 [80] & 95.9 & 6.7 & 8 & 95.7 & 7.3 & 8 & $0.3 \%$ & $0.20[-6.67,7.07]$ \\
\hline Brown et al., 1999_2 [81] & 66.0 & 2.5 & 10 & 63.1 & 2.6 & 10 & $1.6 \%$ & $2.90[0.66,5.14]$ \\
\hline Burke et al., 2001_3 [82] & 62.5 & 2.6 & 5 & 61.5 & 2.7 & 5 & $0.8 \%$ & $1.00[-2.29,4.29]$ \\
\hline Caldow et al., 2015 [83] & 60.7 & 5.6 & 10 & 59.6 & 5.2 & 10 & $0.5 \%$ & $1.10[-3.64,5.84]$ \\
\hline Chycki et al., 2016_1 [84] & 60.6 & 5.2 & 6 & 58.6 & 5.3 & 6 & $0.3 \%$ & $2.00[-3.94,7.94]$ \\
\hline Chycki et al., 2016_2 [84] & 64.3 & 3.2 & 6 & 63.2 & 3.6 & 6 & $0.8 \%$ & $1.10[-2.75,4.95]$ \\
\hline Chycki et al., 2016_3 [84] & 63.2 & 3.6 & 6 & 63.1 & 3.8 & 6 & $0.7 \%$ & $0.10[-4.09,4.29]$ \\
\hline Deyssig et al., 1993_1 [85] & 83.6 & 3.2 & 11 & 76.4 & 3.6 & 11 & $1.5 \%$ & $7.20[4.35,10.05]$ \\
\hline Escalante et al., 2016_1 [86] & 62.0 & 9.7 & 10 & 61.2 & 9.7 & 10 & $0.2 \%$ & $0.80[-7.70,9.30]$ \\
\hline Fahey and Brown 1973_1 [87] & 66.1 & 9.4 & 13 & 63.6 & 8.6 & 13 & $0.2 \%$ & $2.50[-4.43,9.43]$ \\
\hline
\end{tabular}


Table 1. Cont

\begin{tabular}{|c|c|c|c|c|c|c|c|c|}
\hline \multirow[t]{2}{*}{ Study/Group/Year/Reference } & \multicolumn{3}{|c|}{ Post-Training } & \multicolumn{3}{|c|}{ Pre-Training } & \multirow[t]{2}{*}{ Weight } & \multirow{2}{*}{$\begin{array}{l}\text { Mean Difference } \\
\text { Random, 95\% IC }\end{array}$} \\
\hline & Mean & SD & $\mathbf{n}$ & Mean & SD & $\mathrm{n}$ & & \\
\hline Falk et al., 2003_1 [88] & 79.0 & 9.2 & 15 & 77.0 & 8.6 & 15 & $0.3 \%$ & $2.00[-4.37,8.37]$ \\
\hline Falk et al., 2003_2 [88] & 72.7 & 7.7 & 13 & 71.5 & 8.0 & 13 & $0.3 \%$ & $1.20[-4.84,7.24]$ \\
\hline Fyfe et al., 2016 [89] & 60.9 & 5.5 & 8 & 60.1 & 6.0 & 8 & $0.4 \%$ & $0.80[-4.84,6.44]$ \\
\hline Garthe et al., 2013_1 [33] & 61.2 & 8.8 & 21 & 59.4 & 8.9 & 21 & $0.4 \%$ & $1.80[-3.55,7.15]$ \\
\hline Garthe et al., 2013_2 [33] & 61.1 & 6.6 & 18 & 59.9 & 6.7 & 18 & $0.6 \%$ & $1.20[-3.14,5.54]$ \\
\hline Glowacki et al., 2004 [90] & 64.3 & 8.5 & 13 & 61.8 & 8.7 & 13 & $0.3 \%$ & $2.50[-4.11,9.11]$ \\
\hline Gobbo et al., 2013 [91] & 32.4 & 3.5 & 15 & 31.7 & 3.3 & 15 & $2.0 \%$ & $0.70[-1.73,3.13]$ \\
\hline Harber et al., 2004 [92] & 67.3 & 3.4 & 8 & 65.4 & 3.4 & 8 & $1.1 \%$ & $1.90[-1.43,5.23]$ \\
\hline Hoffman et al., 2009_3 [93] & 77.0 & 14.3 & 7 & 76.6 & 13.3 & 7 & $0.1 \%$ & $0.40[-14.07,14.87]$ \\
\hline Hoffman et al., 2012 [94] & 65.6 & 2.2 & 9 & 65.5 & 1.9 & 9 & $3.3 \%$ & $0.10[-1.80,2.00]$ \\
\hline Ihalainen et al., 2018_1 [95] & 62.5 & 6.1 & 37 & 61.3 & 6.1 & 37 & $1.5 \%$ & $1.20[-1.58,3.98]$ \\
\hline Ihalainen et al., 2018_2 [95] & 59.1 & 5.0 & 31 & 58.6 & 5.1 & 31 & $1.9 \%$ & $0.50[-2.01,3.01]$ \\
\hline Joy et al., 2014_1 [39] & 61.0 & 5.6 & 12 & 58.5 & 5.5 & 12 & $0.6 \%$ & $2.50[-1.94,6.94]$ \\
\hline Joy et al., 2016_1 [96] & 65.5 & 6.9 & 11 & 65.3 & 8.1 & 11 & $0.3 \%$ & $0.20[-6.09,6.49]$ \\
\hline Kelly et al, 1998_1 [97] & 78.0 & 11.8 & 9 & 75.2 & 12.0 & 9 & $0.1 \%$ & $2.80[-8.20,13.80]$ \\
\hline Kerksick et al., 2009_1 [98] & 62.2 & 6.1 & 24 & 61.2 & 6.1 & 24 & $1.0 \%$ & $1.00[-2.45,4.45]$ \\
\hline Kerksick et al., 2006_3 [99] & 63.5 & 7.3 & 11 & 63.5 & 8.2 & 11 & $0.3 \%$ & $0.00[-6.49,6.49]$ \\
\hline King et al., 1999_1 [100] & 64.1 & 2.4 & 9 & 61.2 & 2.5 & 9 & $2.3 \%$ & $2.90[0.64,5.16]$ \\
\hline Ko and Choi 2013 [101] & 56.0 & 10.9 & 18 & 55.0 & 10.5 & 18 & $0.2 \%$ & $1.00[-5.99,7.99]$ \\
\hline Kreider et al., 2002_1 [102] & 76.1 & 3.0 & 23 & 75.5 & 3.1 & 23 & $3.8 \%$ & $0.60[-1.16,2.36]$ \\
\hline Lemon et al., 1992_2 [103] & 73.4 & 10.5 & 12 & 73.4 & 10.5 & 12 & $0.2 \%$ & $0.00[-8.40,8.40]$ \\
\hline lo et al., 2011 [104] & 50.8 & 3.3 & 10 & 50.5 & 3.9 & 10 & $1.2 \%$ & $0.30[-2.87,3.47]$ \\
\hline Lowery et al., 2014 [105] & 60.8 & 5.8 & 12 & 58.8 & 6.3 & 12 & $0.5 \%$ & $2.00[-2.85,6.85]$ \\
\hline Mangine et al., 2008_1 [106] & 71.4 & 9.9 & 8 & 69.3 & 10.5 & 8 & $0.1 \%$ & $2.10[-7.90,12.10]$ \\
\hline Mangine et al., 2008_2 [106] & 71.8 & 8.4 & 9 & 69.5 & 8.1 & 9 & $0.2 \%$ & $2.30[-5.32,9.92]$ \\
\hline Mayhew et al., 2009 [107] & 50.5 & 2.5 & 21 & 49.6 & 2.4 & 21 & $5.4 \%$ & $0.90[-0.58,2.38]$ \\
\hline Miller et al., 1984 [108] & 66.6 & 2.2 & 8 & 64.3 & 2.1 & 8 & $2.7 \%$ & $2.30[0.19,4.41]$ \\
\hline Moore et al., 2007 [109] & 64.8 & 6.7 & 12 & 61.6 & 6.9 & 12 & $0.4 \%$ & $3.20[-2.24,8.64]$ \\
\hline Nybo et al., 2010 [9] & 62.8 & 2.7 & 8 & 61.0 & 2.3 & 8 & $2.0 \%$ & $1.80[-0.66,4.26]$ \\
\hline Oliver et al., 2013_1 [52] & 64.2 & 8.5 & 11 & 61.9 & 8.9 & 11 & $0.2 \%$ & $2.30[-4.97,9.57]$ \\
\hline Oliver et al., 2013_2 [52] & 64.3 & 6.8 & 11 & 63.3 & 7.0 & 11 & $0.4 \%$ & $1.00[-4.77,6.77]$ \\
\hline Paoli et al., 2017_1 [110] & 62.4 & 3.3 & 18 & 60.3 & 3.5 & 18 & $2.4 \%$ & $2.10[-0.12,4.32]$ \\
\hline Paoli et al., 2017_2 [110] & 64.2 & 4.9 & 18 & 60.9 & 4.7 & 18 & $1.2 \%$ & $3.30[0.16,6.44]$ \\
\hline Peeters et al., 1999_1 [111] & 75.4 & 12.9 & 14 & 75.3 & 12.8 & 14 & $0.1 \%$ & $0.10[-9.42,9.62]$ \\
\hline Peronnet et al., 1986 [112] & 66.3 & 2.1 & 7 & 64.5 & 2.2 & 7 & $2.4 \%$ & $1.80[-0.45,4.05]$ \\
\hline Pérez-Gómez et al., 2013 [56] & 58.3 & 7.1 & 8 & 57.5 & 7.6 & 8 & $0.2 \%$ & $0.80[-6.41,8.01]$ \\
\hline Saremi et al., 2010_1 [113] & 62.3 & 1.3 & 8 & 60.3 & 1.5 & 8 & $6.3 \%$ & $2.00[0.62,3.38]$ \\
\hline Schneider et al., 2003 [114] & 59.1 & 3.3 & 7 & 57.2 & 3.0 & 7 & $1.1 \%$ & $1.90[-1.40,5.20]$ \\
\hline Schumann et al., 2014_1 [61] & 57.2 & 5.0 & 16 & 55.6 & 4.5 & 16 & $1.1 \%$ & $1.60[-1.70,4.90]$ \\
\hline Schumann et al., 2014_2 [61] & 55.9 & 4.6 & 18 & 54.1 & 4.1 & 18 & $1.5 \%$ & $1.80[-1.05,4.65]$ \\
\hline Slater et al., 2001_1 [115] & 70.1 & 1.0 & 7 & 69.2 & 1.2 & 7 & $8.9 \%$ & $0.90[-0.26,2.06]$ \\
\hline Spence et al., 2011 [64] & 59.1 & 2.5 & 13 & 56.9 & 2.5 & 13 & $3.2 \%$ & $2.20[0.28,4.12]$ \\
\hline Spillane et al., 2016_1 [116] & 62.1 & 5.3 & 11 & 61.8 & 4.3 & 11 & $0.7 \%$ & $0.30[-3.73,4.33]$ \\
\hline
\end{tabular}


Table 1. Cont.

\begin{tabular}{|c|c|c|c|c|c|c|c|c|}
\hline \multirow[t]{2}{*}{ Study/Group/Year/Reference } & \multicolumn{3}{|c|}{ Post-Training } & \multicolumn{3}{|c|}{ Pre-Training } & \multirow[t]{2}{*}{ Weight } & \multirow{2}{*}{$\begin{array}{l}\text { Mean Difference } \\
\text { Random, } 95 \% \text { IC }\end{array}$} \\
\hline & Mean & SD & $\mathrm{n}$ & Mean & SD & $\mathbf{n}$ & & \\
\hline Taylor et al., 2011_1 [117] & 66.2 & 8.3 & 15 & 65.7 & 8.8 & 15 & $0.3 \%$ & $0.50[-5.62,6.62]$ \\
\hline Thorstensson et al., 1976 [118] & 65.0 & 1.2 & 14 & 62.8 & 1.3 & 14 & $13.9 \%$ & $2.20[1.27,3.13]$ \\
\hline Tinsley et al., 2017_1 [119] & 58.7 & 8.0 & 18 & 56.4 & 9.3 & 18 & $0.4 \%$ & $2.30[-3.37,7.97]$ \\
\hline Volek et al., 2004 [120] & 70.6 & 5.8 & 9 & 67.2 & 5.6 & 9 & $0.4 \%$ & $3.40[-1.87,8.67]$ \\
\hline Yan et al., 2016_1 [121] & 58.8 & 4.7 & 8 & 57.9 & 5.0 & 8 & $0.5 \%$ & $0.90[-3.86,5.66]$ \\
\hline Yan et al., 2016_2 [121] & 61.0 & 7.7 & 9 & 59.5 & 7.3 & 9 & $0.2 \%$ & $1.50[-5.43,8.43]$ \\
\hline Yan et al., 2016_3 [121] & 59.3 & 6.6 & 8 & 57.6 & 6.5 & 8 & $0.3 \%$ & $1.70[-4.72,8.12]$ \\
\hline Total $(95 \%$ CI $)$ & & & 810 & & & 810 & $100 \%$ & $1.65[1.28,2.01]$ \\
\hline Heterogeneity: $\mathrm{Tau}^{2}=0.00 ; \mathrm{Cl}$ & 3.79, & 84 & $=1.0$ & $=0 \%$ & & for o & all effect: & $=8.91(p<0.00001)$ \\
\hline
\end{tabular}

\subsubsection{The Resistance Training Characteristics}

The types of training performed were circuit training (n groups: $F F M=1 ; L M M=2$ ), full body (n groups: $\mathrm{FFM}=40 ; \mathrm{LMM}=31 ; \mathrm{SMM}=4$ ), traditional split routine (n groups: $\mathrm{FFM}=34 ; \mathrm{LMM}=20$; SMM $=7$ ), lower limbs (n groups: $L M M=3$ ), and physical military training (n groups: $F F M=2$ ) and not specified (n groups: $F F M=5 ; \mathrm{LMM}=9$ ) for FFM. The training sessions lasted from 4 to 24 weeks $(\mathrm{FFM}=10.4 \pm 5.41 ; \mathrm{LMM}=9.37 \pm 4.53 ; \mathrm{SMM}=10.5 \pm 4.01)$ with a weekly frequency of between 2 and 6 days $(\mathrm{FFM}=3.5 \pm 0.84 ; \mathrm{LMM}=3.2 \pm 0.81 ; \mathrm{SMM}=3.3 \pm 0.94)$. The resistance training sessions were carried out with between 1 and 20 exercises (FFM $=7.9 \pm 3.13 ; \mathrm{LMM}=7.3 \pm 3.67 ; \mathrm{SMM}=7.0 \pm$ $1.48), 1$ to 50 sets per exercise $(\mathrm{FFM}=16.7 \pm 11.8 ; \mathrm{LMM}=14.0 \pm 10.1 ; \mathrm{SMM}=19.1 \pm 11.1)$ and 2 to 15 repetitions per set (FFM $=7.8 \pm 3.0 ; \mathrm{LMM}=9.1 \pm 1.9 ; \mathrm{SMM}=8.5 \pm 0.7)$ with intensities of 50 and $89 \%$ of $1 \mathrm{RM}(\mathrm{FFM}=78.8 \pm 5.8 ; \mathrm{LMM}=74.6 \pm 8.6 ; \mathrm{SMM}=80.8 \pm 5.8)$.

\subsection{Heterogeneity and Risk of Bias}

Risk-of-bias assessment is shown in Figure 2. Overall, the risk of bias was low in the studies, the overall median scores were high, 10/12 points.

In addition, heterogeneity was not found for changes in hypertrophy for overall effects $(Z=-0.078$; $p=0.94)$. In addition, heterogeneity was not observed when studies were divided according to FFM $(Z=-0.003 ; p=0.99) ; \operatorname{LMM}(Z=-0.25, p=0.80)$ and SMM $(Z=-0.58, p=0.56)$.

\subsection{Meta-Analyses}

\subsubsection{Effects of Training on Hypertrophy}

The results of the overall effects on muscle mass (FFM + LMM + SMM) before and after the resistance training programme showed significant improvement between pre- and post-test (n participants $\left.=1916 ; 1.53 \mathrm{~kg} 95 \% \mathrm{CI}[1.30,1.76], p<0.001 ; \mathrm{I}^{2}=0 \%, p=1.00\right)$.

\subsubsection{Subgroup Analysis}

RT evoked a significant increase of FFM (1.56 kg 95\% CI [1.23, 1.89]), of LMM (1.65 kg 95\% CI $[1.28,2.01])$ and of SMM $(1.11 \mathrm{~kg} 95 \%$ CI $[0.52,1.71])$ (Table 1). However, non-significant differences were observed between the different variables used to describe hypertrophy used.

When the studies were divided using the training status of participants, non- significant differences were observed between trained and untrained participants after the resistance training programme (Figure 3). However, when comparing pre-post intervention results for each way of measuring hypertrophy, both groups present significant increases for FFM (untrained: $1.54 \mathrm{~kg} 95 \% \mathrm{CI}[1.12,1.96]$, trained: $0.98 \mathrm{~kg} 95 \%$ CI [0.17, 1.79]), for LMM (untrained: $1.70 \mathrm{~kg} 95 \%$ CI [1.04, 2.36], trained: $1.09 \mathrm{~kg}$ 
95\% CI [0.36, 1.81]), except for the SMM (untrained: $1.22 \mathrm{~kg} 95 \%$ CI $[0.38,2.06]$, trained: $0.66 \mathrm{~kg} 95 \% \mathrm{CI}$ $[-0.39,1.72])$.

\section{Fat Free Mass}

\begin{tabular}{|c|}
\hline Study or Subgroup \\
\hline Untrained \\
\hline Ahtiainen et al. 2011 \\
\hline Arciero et al. 2001_1 \\
\hline Arciero et al. 2001_2 \\
\hline Buresh et al 2009_1 \\
\hline Buresh et al 2009_2 \\
\hline Chromiak et al. 2004_1 \\
\hline Chromiak et al. 2004_2 \\
\hline Carraguer et al. 2000_1 \\
\hline Carraguer et al. 2000_2 \\
\hline Chahramanloo et al. 2009 \\
\hline Hong et al. 2014_1 \\
\hline Hong et al. 2014 \\
\hline Hu et al. 2009 \\
\hline Jang et al. 2017_1 \\
\hline Kim et al. 2014_1 \\
\hline Kramer et al. 2009_2 \\
\hline Lemmer et al. 2001 \\
\hline Lockwood et al 2017_1 \\
\hline Lukaski et al. 1996_1 \\
\hline Lukaski et al. 1996_2 \\
\hline Lukaski et al. 1996_3 \\
\hline McCarthy et al. 1995 \\
\hline Radaelli et al. 2015_1 \\
\hline Radaelli et al. 2015_2 \\
\hline Radaelli et al. 2015_3 \\
\hline Roberts et al. 2007_2 \\
\hline Schumann et al. 2014_1 \\
\hline Schumann et al. 2014_2 \\
\hline Shelmadine et al. 2009_2 \\
\hline Snijders et al. 2015_1 \\
\hline Spence et al. 2011 \\
\hline Spillane et al. 2009_3 \\
\hline Spillane et al. 2011_ 1 \\
\hline Terzis et al. 2008_i \\
\hline Terzis et al. 2008_z \\
\hline Thomson et al. 2009_2 \\
\hline Willoughby et al. 2007_1 \\
\hline Zamani et al. 2017_1 \\
\hline Zamani et al. 2017_2 \\
\hline Zamani et al. 2017_3 \\
\hline \\
\hline
\end{tabular}

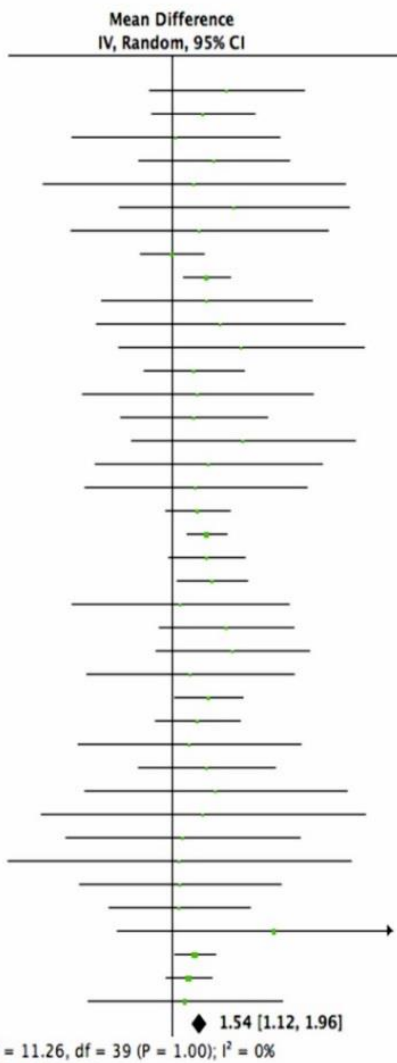

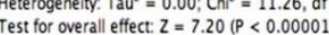

Trained

Alvehus et al. 2014

Bang et al. 2017_1

Bartolomei et al. 2016_1

Bartolomei et al. 2016_2

Bartolomei et al. 2018_1

Bartolomei et al. 2018_2

Colquhoun et al. 2017_1

Crewther et al. 2013

Crewther et al. 2013

Fu et al. 2017_1

Fu et al. 2017- 2017

Carthe et al. 2013_1

Carthe et al. 2013

Joy et al. 2014

Kreipke et al. 2015_1

Mazzerti et al. 2000

Mazzetti et al. 2000

Naczeiro et al. 2017

Oliver et al. 2013_1

Oliver et al. 2013

Ormsbee et al. 2012

Ormsbee et al. 2013

Ribeiro et al. 2015 1

Ribeiro et al. 2015_2

Ribeiro et al. 2015_2
Rogerson et al. 2007_z

Tomljanović et al. 2011.

Womljanovic et al. 2011_

Wilbonr, et al. 2010_2
Willoughby et al. 2007_2

Willoughby et al. 2007
Subtotal $(95 \% \mathrm{CI})$

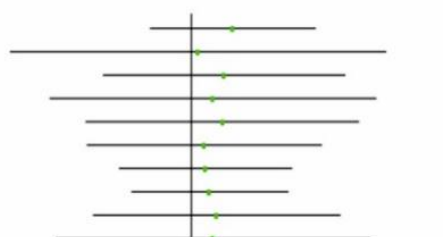

Heterogeneity: Taü $^{2}=0.00 ; \mathrm{Ch}^{2}=2.41$,
Test for overall effect: $Z=2.36(P=0.02)$

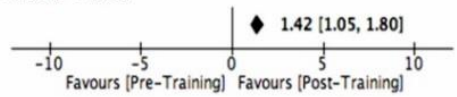

Total $(95 \% \mathrm{Cl})$

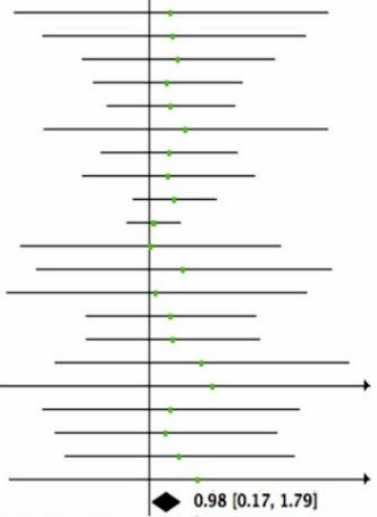

Heterogeneity: $\mathrm{Tau}^{2}=0.00 ; \mathrm{Ch}^{2}=15.15, \mathrm{df}=69(\mathrm{P}=1.00) ; \mathrm{I}^{2}=0 x$

Test for overall effect: $Z=7.48(P<0.00001)$

Test for subaroup differences: $\mathrm{Ch}^{2}=1.48, \mathrm{df}=1(P=0.22), \mathrm{I}^{2}=32.4 \%$

\section{Lean Muscle Mass}

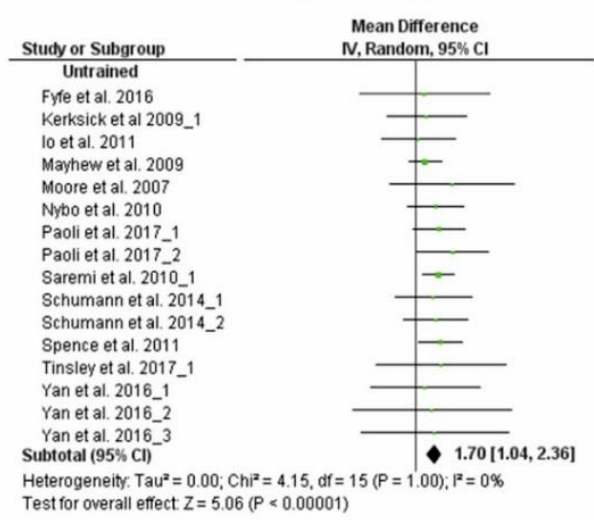

Test for overall effect $Z=5.06(P<0.0000$

\section{Trained}

Alcaraz et al. 2011_1

Alcaraz et al. 2011_2

Alvehus et al. 2014

Andre et al. 2016

Ara et al. 2006

Burke et al. 2001_3

Caldowet al. 2015

Chycki et a. 2016_1

Chycki et al. 2016-2

Escalante et al. 2016

Garthe et al. 2013_1

Garthe et al. 2013-

Garthe et al. $2013-2$

Hoffman et al. 2009

Hoffman et al. 2009_3

Hoffman et al. 2012-1

Ihalainen et al. $2017_{-} 2$

Joy et al. 2013_1

Kerksick et al. 2006

Kerksick et al. 2006

Lencing

Mangine et al 2008-

Miller et al. 1984

Oliver et al. 2013

Oliver et al. 2013_1

Spillane et al al. 2016

Subtotal $(95 \% \mathrm{Cl})$

Heterogeneity Tau" =

$\mathrm{Ch}^{2}=4.45, \mathrm{df}=25(P=1.00) ; \mathrm{P}^{2}=0 \%$

Test for overall effect $Z=2.93(P=0.003)$

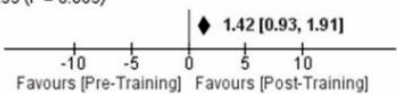

Total $(95 \% \mathrm{Cl})$

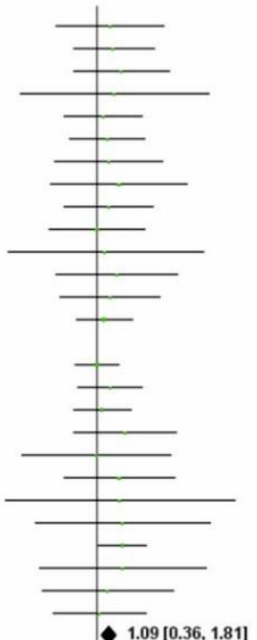

Favours [Pre-Training] Favours [Post-Training]

Heterogeneity. $\mathrm{Tau}^{2}=0.00 ; \mathrm{Ch}^{2}=10.10, \mathrm{df}=41 \quad(P=1.00) ; \mathrm{P}^{2}=0$ \%

Test for overall effect $Z=5.72(P<0.00001)$

Test for subaroup differences: $\mathrm{Ch}^{2}=1.49, \mathrm{df}=1(\mathrm{P}=0.22), \mathrm{P}^{2}=33.1 \%$

\section{Skeletal Muscle Mass}

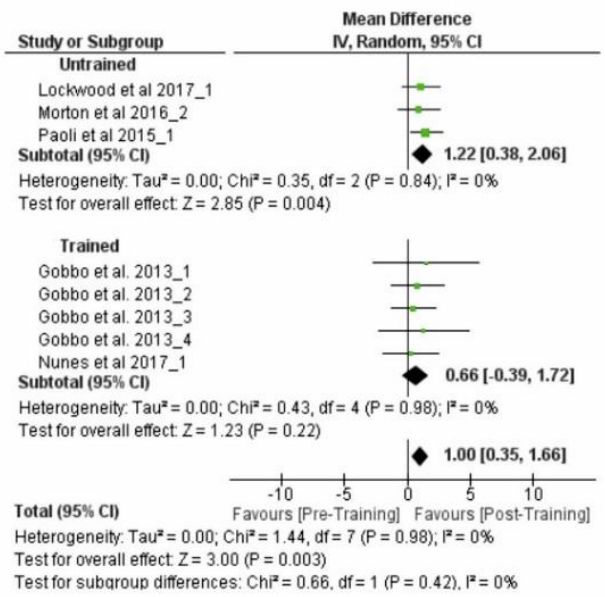

Figure 3. Mean differences exploration comparing the training effect. 
Performing a more detailed analysis of the training status on FFM, we found that the categories sedentary/untrained and physically active but no experience in RT, have higher and more significant values than the moderately trained (RT up to 1 year/intermediate, RT experience up to 2 years/intermediate, and RT experience up to 3 years/intermediate). Additionally, the highest values achieved are those obtained by the more experienced subjects, RT experience of 4 years or more/advanced (see Figure 4). However, no statistically significant differences between categories were observed $\left(\chi^{2}=5.79 ; p=0.33\right)$.

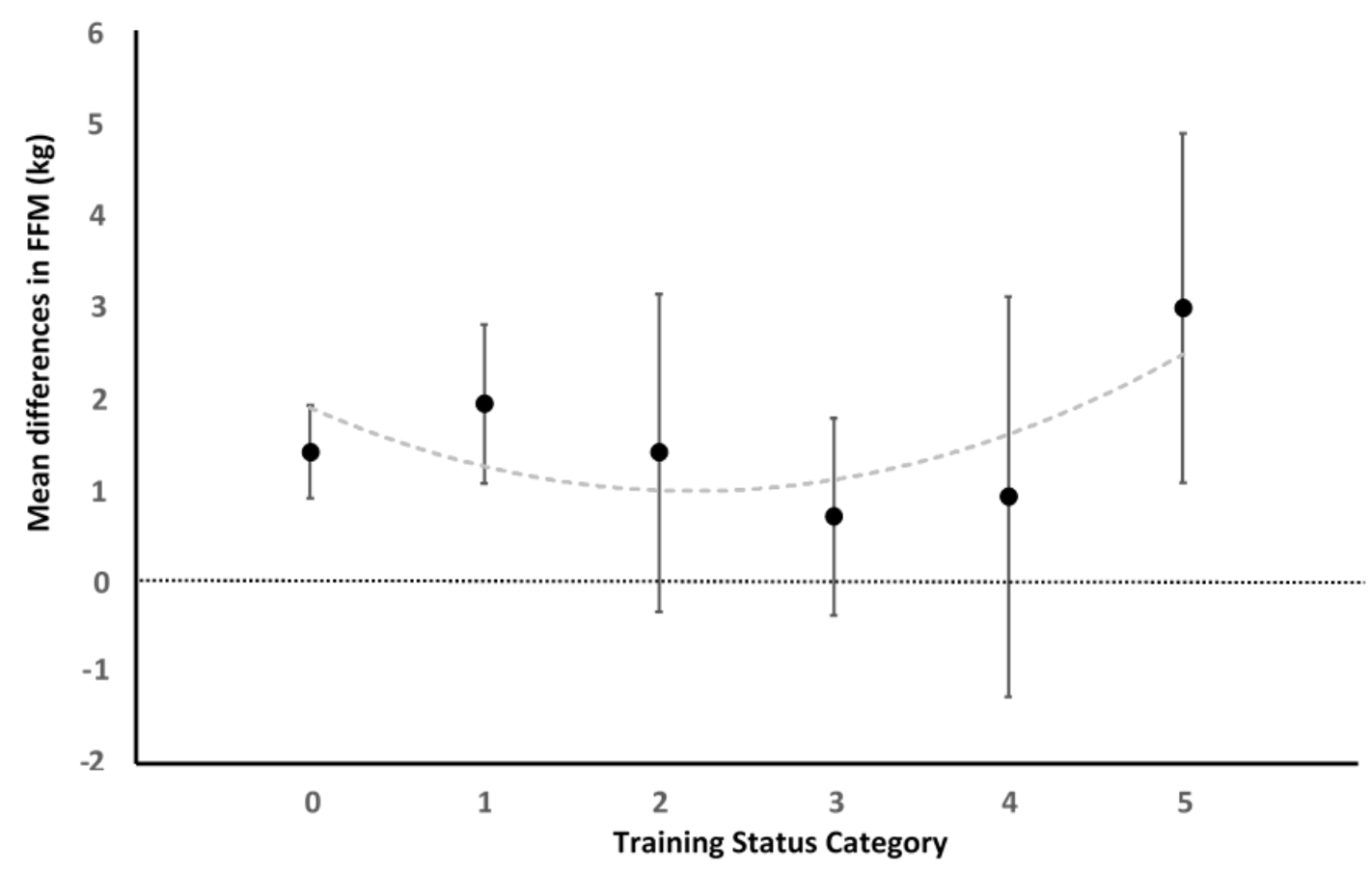

Figure 4. Mean differences exploration comparing the training status by categories. Sedentary $=0$ : $1.39 \mathrm{~kg} \mathrm{95 \%} \mathrm{CI} \mathrm{[0.89,} \mathrm{1.90],} p<0.001 ; \mathrm{I}^{2}=0 \%, p=0.89 ;$ untrained $=1: 1.92 \mathrm{~kg} \mathrm{95 \%} \mathrm{CI}[1.05,2.78]$, $p<0.001 ; \mathrm{I}^{2}=0 \%, p=1.00 ; \mathrm{Up}$ to one year experience $=2: 1.39 \mathrm{~kg} 95 \% \mathrm{CI}[-0.35,3.12], p=0.12 ; \mathrm{I}^{2}=$ $0 \%, p=1.00$; Up to two year experience $=3: 0.69 \mathrm{~kg} 95 \% \mathrm{CI}[-0.39,1.76], p=0.21 ; \mathrm{I}^{2}=0 \%, p=1.00 ; \mathrm{Up}$ to tree year experience $=4: 0.9 \mathrm{~kg} 95 \% \mathrm{CI}[-1.28,3.09], p=0.42 ; \mathrm{I}^{2}=0 \%, p=1.00 ;$ RT experience of 4 years or more $=5: 2.96 \mathrm{~kg} 95 \% \mathrm{CI}[1.06,4.87], p=0.002 ; \mathrm{I}^{2}=41 \%, p=0.08$.

\subsection{Meta-Regression}

The results from the meta-regression model are presented in Table 2. Considering the overall effects, and taking into account the participant characteristics, none of our covariates explained any effect on changes in muscle mass. Regarding the training characteristics, the only significant variable that explains the variance of the hypertrophy was the sets per workout, showing a significant negative interaction (MD; estimate: 1.85, 95\% CI [1.45, 2.25], $p<0.001$; moderator: -0.03 95\% CI [-0.05, $-0.00] p=0.041$ ). When the effect of RT on changes in hypertrophy was evaluated with muscle mass assessment stratified into three subgroups (FFM, LMM and SMM) there were no covariates that explained any of the variance in the change in hypertrophy. 
Table 2. Metaregression analisys taking in to account de participants and training characteristics, at the begin the study.

\begin{tabular}{|c|c|c|c|c|c|c|c|c|c|c|c|c|c|}
\hline \multirow{2}{*}{ Model } & \multirow{2}{*}{$\begin{array}{c}\text { n Studies/ } \\
\text { Groups }\end{array}$} & Estimate & Lower & Upper & $p$ & Estimate & Lower & Upper & $p$ & $\tau^{2}$ & Adj. $R^{2}$ & $\mathrm{I}^{2}$ & $p$ \\
\hline & & \multicolumn{4}{|c|}{ Intrcpt } & \multicolumn{4}{|c|}{ Moderator coeff. } & \multicolumn{4}{|c|}{ Heterogeneity } \\
\hline \multicolumn{14}{|l|}{ Overall Effects } \\
\hline No covariables & 158 & 1.530 & 1.300 & 1.753 & $<0.001$ & & & & & $0(\mathrm{SE}=0.13)$ & & $0 \%$ & 1.000 \\
\hline \multicolumn{14}{|l|}{ Participants Characteristics } \\
\hline Age (years) & 142 & 0.943 & -0.962 & 2.849 & 0.332 & 0.02 & -0.06 & 0.10 & 0.58 & $0(\mathrm{SE}=0.15)$ & NA $\%$ & $0 \%$ & 1.000 \\
\hline Weight $(\mathrm{kg})$ & 150 & 1.138 & -1.822 & 4.098 & 0.451 & 0.00 & -0.03 & 0.04 & 0.82 & $0(\mathrm{SE}=0.14)$ & NA $\%$ & $0 \%$ & 1.000 \\
\hline Height (m) & 126 & -0.014 & -6.032 & 6.004 & 0.996 & 0.80 & -2.58 & 4.18 & 0.64 & $0(\mathrm{SE}=0.15)$ & NA $\%$ & $0 \%$ & 1.000 \\
\hline Training Status & 119 & 1.547 & 1.167 & 1.927 & $<0.001$ & -0.16 & -0.40 & 0.08 & 0.19 & $0(\mathrm{SE}=0.18)$ & NA $\%$ & $0 \%$ & 1.000 \\
\hline \multicolumn{14}{|l|}{ Training Characteristics } \\
\hline Study Durations (weeks) & 156 & 1.428 & 0.896 & 1.960 & $<0.001$ & 0.01 & -0.04 & 0.06 & 0.69 & $0(\mathrm{SE}=0.13)$ & NA $\%$ & $0 \%$ & 1.000 \\
\hline Sessions & 152 & 1.492 & 0.939 & 2.045 & $<0.001$ & 0.00 & -0.02 & 0.02 & 0.89 & $0(\mathrm{SE}=0.13)$ & NA $\%$ & $0 \%$ & 1.000 \\
\hline Days per week & 149 & 1.528 & 0.502 & 2.553 & 0.004 & -0.01 & -0.31 & 0.30 & 0.97 & $0(\mathrm{SE}=0.14)$ & NA $\%$ & $0 \%$ & 1.000 \\
\hline $\mathrm{n}$ exercises per workout & 141 & 1.423 & 0.640 & 2.207 & $<0.001$ & 0.01 & -0.09 & 0.11 & 0.87 & $0(\mathrm{SE}=0.16)$ & NA $\%$ & $0 \%$ & 1.000 \\
\hline Rest between exercise (min) & 121 & 1.336 & 0.646 & 2.025 & $<0.001$ & 0.05 & -0.23 & 0.32 & 0.74 & $0(\mathrm{SE}=0.16)$ & NA $\%$ & $0 \%$ & 1.000 \\
\hline n set per workout & 123 & 1.853 & 1.453 & 2.253 & $<0.001$ & -0.03 & -0.05 & -0.00 & 0.04 & $0(\mathrm{SE}=0.14)$ & NA $\%$ & $0 \%$ & 1.000 \\
\hline range repetitions & 123 & 1.257 & 0.486 & 2.029 & 0.001 & 0.02 & -0.07 & 0.12 & 0.61 & $0(\mathrm{SE}=0.17)$ & NA $\%$ & $0 \%$ & 1.000 \\
\hline Training duration (min) & 35 & 1.548 & -0.248 & 3.344 & 0.091 & 0.00 & -0.03 & 0.03 & 0.75 & $0(\mathrm{SE}=0.40)$ & NA $\%$ & $0 \%$ & 1.000 \\
\hline Average Intensity (\%1RM) & 89 & 2.353 & -1.383 & 6.089 & 0.217 & -0.01 & -0.06 & 0.04 & 0.63 & $0(\mathrm{SE}=0.22)$ & NA $\%$ & $0 \%$ & 1.000 \\
\hline \multicolumn{14}{|l|}{ Fat Free Mass } \\
\hline No covariables & 82 & 1.550 & 1.223 & 1.886 & $<0.001$ & & & & & $0(\mathrm{SE}=1.91)$ & & $0 \%$ & 1.000 \\
\hline \multicolumn{14}{|l|}{ Participants Characteristics } \\
\hline Age (years) & 77 & 1.116 & -1.534 & 3.766 & 0.409 & 0.02 & -0.09 & 0.13 & 0.74 & $0(\mathrm{SE}=1.95)$ & NA $\%$ & $0 \%$ & 1.000 \\
\hline Weight $(\mathrm{kg})$ & 80 & -0.183 & -4.371 & 4.006 & 0.932 & 0.02 & -0.03 & 0.08 & 0.42 & $0(\mathrm{SE}=0.21)$ & NA $\%$ & $0 \%$ & 1.000 \\
\hline Height (m) & 67 & -1.960 & -18.842 & 14.930 & 0.82 & 1.96 & -7.55 & 11.46 & 0.69 & $0(\mathrm{SE}=0.21)$ & NA $\%$ & $0 \%$ & 1.000 \\
\hline
\end{tabular}


Table 2. Cont

\begin{tabular}{|c|c|c|c|c|c|c|c|c|c|c|c|c|c|}
\hline \multirow{2}{*}{ Model } & \multirow{2}{*}{$\begin{array}{c}\text { n Studies/ } \\
\text { Groups }\end{array}$} & Estimate & Lower & Upper & $p$ & Estimate & Lower & Upper & $p$ & $\tau^{2}$ & Adj. $R^{2}$ & $\mathrm{I}^{2}$ & $p$ \\
\hline & & \multicolumn{4}{|c|}{ Intrcpt } & \multicolumn{4}{|c|}{ Moderator coeff. } & \multicolumn{4}{|c|}{ Heterogeneity } \\
\hline Training Status & 70 & 1.530 & 1.082 & 1.978 & $<0.001$ & -0.13 & -0.41 & 0.15 & 0.37 & $0(\mathrm{SE}=0.24)$ & NA $\%$ & $0 \%$ & 1.000 \\
\hline \multicolumn{14}{|l|}{ Training Characteristics } \\
\hline Study Durations (weeks) & 80 & 1.314 & 0.534 & 2.094 & $<0.001$ & 0.02 & -0.05 & 0.10 & 0.50 & $0(\mathrm{SE}=0.20)$ & NA $\%$ & $0 \%$ & 1.000 \\
\hline Sessions & 76 & 1.172 & 0.297 & 2.047 & 0.009 & 0.01 & -0.01 & 0.04 & 0.35 & $0(\mathrm{SE}=0.20)$ & NA $\%$ & $0 \%$ & 1.000 \\
\hline Days per week & 76 & 1.154 & -0.325 & 2.633 & 0.126 & 0.11 & -0.30 & 0.53 & 0.59 & $0(\mathrm{SE}=0.20)$ & NA $\%$ & $0 \%$ & 1.000 \\
\hline $\mathrm{n}$ exercises per workout & 73 & 1.280 & 0.238 & 2.321 & 0.016 & 0.04 & -0.10 & 0.17 & 0.59 & $0(\mathrm{SE}=0.21)$ & NA $\%$ & $0 \%$ & 1.000 \\
\hline Rest between exercise (min) & 56 & 2.086 & 0.096 & 4.075 & 0.04 & -0.36 & -1.40 & 0.68 & 0.50 & $0(\mathrm{SE}=0.27)$ & NA $\%$ & $0 \%$ & 1.000 \\
\hline n set per workout & 66 & 1.739 & 1.181 & 2.296 & $<0.001$ & -0.01 & -0.05 & 0.02 & 0.49 & $0(\mathrm{SE}=0.20)$ & NA $\%$ & $0 \%$ & 1.000 \\
\hline range repetitions & 72 & 1.199 & 0.335 & 2.064 & 0.007 & 0.05 & -0.07 & 0.17 & 0.38 & $0(\mathrm{SE}=0.20)$ & NA $\%$ & $0 \%$ & 1.000 \\
\hline Training duration (min) & 26 & 1.467 & -1.415 & 4.349 & 0.318 & 0.01 & -0.05 & 0.06 & 0.79 & $0(\mathrm{SE}=0.47)$ & NA $\%$ & $0 \%$ & 1.000 \\
\hline Average Intensity (\%1RM) & 45 & 3.993 & -3.027 & 11.012 & 0.265 & -0.03 & -0.12 & 0.06 & 0.48 & $0(\mathrm{SE}=0.26)$ & NA $\%$ & $0 \%$ & 1.000 \\
\hline \multicolumn{14}{|l|}{ Lean Muscle Mass } \\
\hline No covariables & 65 & 1.644 & 1.275 & 2.013 & $<0.0001$ & & & & & $0.03(\mathrm{SE}=0.24)$ & & $1 \%$ & 0.999 \\
\hline \multicolumn{14}{|l|}{ Participants Characteristics } \\
\hline Age (years) & 54 & 1.020 & -1.964 & 4.005 & 0.5028 & 0.02 & -0.10 & 0.14 & 0.73 & $0.03(\mathrm{SE}=0.33)$ & NA $\%$ & $1 \%$ & 0.996 \\
\hline Weight $(\mathrm{kg})$ & 60 & 4.870 & 0.002 & 9.739 & 0.0499 & -0.04 & -0.10 & 0.02 & 0.18 & $0(\mathrm{SE}=0.25)$ & NA $\%$ & $0 \%$ & 1.000 \\
\hline Height (m) & 49 & 0.107 & -6.453 & 6.666 & 0.9746 & 0.73 & -2.94 & 4.40 & 0.70 & $0(\mathrm{SE}=0.33)$ & NA $\%$ & $0 \%$ & 1.000 \\
\hline Training Status & 41 & 1.584 & 0.721 & 2.446 & 0.0003 & -0.15 & -0.66 & 0.36 & 0.57 & $0(\mathrm{SE}=0.44)$ & NA $\%$ & $0 \%$ & 1.000 \\
\hline \multicolumn{14}{|l|}{ Training Characteristics } \\
\hline Study Durations (weeks) & 65 & 1.701 & 0.881 & 2.521 & $<0.0001$ & -0.01 & -0.08 & 0.07 & 0.87 & $0.04(\mathrm{SE}=0.26)$ & 0.00 & $2 \%$ & 0.999 \\
\hline Sessions & 65 & 1.860 & 1.087 & 2.633 & $<0.0001$ & -0.01 & -0.03 & 0.02 & 0.53 & $0.05(\mathrm{SE}=0.26)$ & 0 & $2 \%$ & 0.999 \\
\hline Days per week & 62 & 1.996 & -0.184 & 4.175 & 0.0727 & -0.13 & -0.84 & 0.58 & 0.72 & $0(\mathrm{SE}=0.24)$ & NA $\%$ & $0 \%$ & 1.000 \\
\hline n exercises per workout & 57 & 1.661 & 0.289 & 3.032 & 0.0176 & -0.01 & -0.18 & 0.16 & 0.92 & $0(\mathrm{SE}=0.45)$ & NA $\%$ & $0 \%$ & 1.000 \\
\hline Rest between exercise (min) & 54 & 1.458 & 0.279 & 2.636 & 0.0153 & 0.06 & -0.37 & 0.50 & 0.78 & $0(\mathrm{SE}=0.26)$ & NA $\%$ & $0 \%$ & 1.000 \\
\hline
\end{tabular}


Table 2. Cont

\begin{tabular}{|c|c|c|c|c|c|c|c|c|c|c|c|c|c|}
\hline \multirow{2}{*}{ Model } & \multirow{2}{*}{$\begin{array}{l}\text { n Studies/ } \\
\text { Groups }\end{array}$} & Estimate & Lower & Upper & $p$ & Estimate & Lower & Upper & $p$ & $\tau^{2}$ & Adj. $R^{2}$ & $I^{2}$ & $p$ \\
\hline & & \multicolumn{4}{|c|}{ Intrcpt } & \multicolumn{4}{|c|}{ Moderator coeff. } & \multicolumn{4}{|c|}{ Heterogeneity } \\
\hline n set per workout & 46 & 2.082 & 1.417 & 2.748 & $<0.0001$ & -0.04 & -0.09 & 0.01 & 0.09 & $0(\mathrm{SE}=0.29)$ & NA $\%$ & $0 \%$ & 1.000 \\
\hline range repetitions & 44 & 0.601 & -1.874 & 3.076 & 0.6341 & 0.08 & -0.17 & 0.33 & 0.54 & $0(\mathrm{SE}=0.43)$ & $\mathrm{NA} \%$ & $0 \%$ & 1.000 \\
\hline Training duration (min) & 9 & 1.256 & -1.867 & 4.379 & 0.4306 & 0.01 & -0.03 & 0.05 & 0.75 & $0(\mathrm{SE}=1.61)$ & NA\% & $0 \%$ & 0.998 \\
\hline Average Intensity (\%1RM) & 41 & 0.807 & -3.984 & 5.598 & 0.7413 & 0.01 & -0.05 & 0.07 & 0.77 & $0(\mathrm{SE}=0.54)$ & NA\% & $0 \%$ & 1.000 \\
\hline \multicolumn{14}{|l|}{ Skeletal Mascle Mass } \\
\hline No covariables & 11 & 1.11 & 0.519 & 1.709 & $<0.001$ & & & & & $0(\mathrm{SE}=0.41)$ & & $0 \%$ & 0.920 \\
\hline \multicolumn{14}{|l|}{ Participants Characteristics } \\
\hline Age (years) & 11 & 1.5 & -7.901 & 10.901 & 0.755 & -0.017 & -0.424 & 0.391 & 0.936 & $0(\mathrm{SE}=0.48)$ & $\mathrm{NA} \%$ & $0 \%$ & 0.873 \\
\hline Weight (kg) & 10 & -1.792 & -15.819 & 12.236 & 0.802 & 0.038 & -0.152 & 0.227 & 0.695 & $0(\mathrm{SE}=0.56)$ & NA $\%$ & $0 \%$ & 0.863 \\
\hline Height (m) & 10 & -3.23 & -41.849 & 35.385 & 0.870 & 2.37 & -19.223 & 23.964 & 0.830 & $0(\mathrm{SE}=0.55)$ & $\mathrm{NA} \%$ & $0 \%$ & 0.853 \\
\hline Training Status & 8 & 2.448 & 0.418 & 4.478 & 0.018 & -0.893 & -2.259 & 0.474 & 0.201 & $0(\mathrm{SE}=0.51)$ & NA $\%$ & $0 \%$ & 0.956 \\
\hline \multicolumn{14}{|l|}{ Training Characteristics } \\
\hline Study Durations (weeks) & 11 & 0.885 & -0.935 & 2.704 & 0.341 & 0.023 & -0.148 & 0.194 & 0.794 & $0(\mathrm{SE}=0.44)$ & NA $\%$ & $0 \%$ & 0.878 \\
\hline Sessions & 11 & 1.941 & -2.455 & 6.337 & 0.387 & -0.027 & -0.171 & 0.116 & 0.710 & $0(\mathrm{SE}=0.48)$ & NA $\%$ & $0 \%$ & 0.884 \\
\hline Days per week & 11 & 1.824 & -0.571 & 4.219 & 0.136 & -0.217 & -0.926 & 0.492 & 0.549 & $0(\mathrm{SE}=0.44)$ & $\mathrm{NA} \%$ & $0 \%$ & 0.900 \\
\hline $\mathrm{n}$ exercises per workout & 11 & 3.197 & 0.25 & 6.143 & 0.034 & -0.305 & -0.728 & 0.118 & 0.157 & $0(\mathrm{SE}=0.44)$ & NA $\%$ & $0 \%$ & 0.980 \\
\hline Rest between exercise (min) & 11 & 0.719 & -0.657 & 2.095 & 0.306 & 0.141 & -0.301 & 0.583 & 0.532 & $0(\mathrm{SE}=0.44)$ & $\mathrm{NA} \%$ & $0 \%$ & 0.902 \\
\hline $\mathrm{n}$ set per workout & 11 & 1.489 & 0.304 & 2.674 & 0.014 & -0.022 & -0.082 & 0.038 & 0.474 & $0(\mathrm{SE}=0.45)$ & NA $\%$ & $0 \%$ & 0.910 \\
\hline range repetitions & 7 & 3.056 & -8.385 & 14.497 & 0.601 & -0.247 & -1.593 & 1.1 & 0.720 & $0(\mathrm{SE}=0.72)$ & NA $\%$ & $0 \%$ & 0.968 \\
\hline
\end{tabular}

Training duration (min)

Average Intensity (\%1RM) 


\section{Discussion}

The main finding of the present study is that RT significantly increases muscle mass, in average $(\mathrm{FFM}=1.56 / \mathrm{LMM}=1.65 / \mathrm{SMM}=1.11 \mathrm{~kg})$, by about $1.5 \mathrm{~kg}$ with a wide range of heterogeneity (from 0 to $7.2 \mathrm{~kg}$ ). These results were obtained including studies with interventions durations ranging from 2 weeks to one year. The present meta-analysis is, to date, the largest in terms of RT interventions on full body muscle mass gain. Most meta-analyses that have analysed the effect of RT and characteristics of participants on muscle mass gains have only included works with protein supplementation [6,122-129] or have analysed single variables of the training load isolated, as for example, training frequency [130-132], intensity or training periodization [133]. This segmentation has meant that many of the works that have focused solely on these analyses have been left out. However, as stated by Morton et al. [6], performance of RT alone is the much more potent stimulus, accounting for a substantially greater portion of the variance in RT-induced gain in muscle mass. Therefore, in order to have the largest number of studies that met all the inclusion criteria, the meta-analyses should focus on the variables of the training or the characteristics of the participants that have the greatest effect on muscle mass. The most important findings are that RT is an effective method to develop hypertrophy, regardless of the variable used to quantify it, as well as the training characteristics (intensity, volume, weekly training frequency, etc.), with significant increases of $1.6 \mathrm{~kg}$ in FFM; of $1.7 \mathrm{~kg}$ in LMM; and $1.1 \mathrm{~kg}$ in muscle mass (MM). These results are slightly higher than those found in the meta-regression of Morton et al. [6], increasing $1.1 \mathrm{~kg}$ of FFM when supplementation is not included and $1.4 \mathrm{~kg}$ in the FFM when supplementation is included. This may be due to the fact that only studies including intervention with nutritional supplements were considered in Morton's work, and that the no-supplementation results were derived from the control groups of these studies. This considerably reduced their sample and may explain their possible underestimation of muscle mass. On the other hand, although there is no clear moderating factor, it is observed that there are certain variables such as the training status (the untrained have greater gains in hypertrophy), as well as other variables related to the training load, mainly the number of sets per workout, which explain a greater gain in this variable when the number is relatively low, always within a threshold. However, due to the lack of explanation of the variance from the variables of the training load analyzed, it has not been possible to fulfil the objective of creating a mathematical model for the estimation of the level of human muscle hypertrophy in men.

\subsection{Participant Characteristics}

Regarding the characteristics of the participants, no variable has been shown to moderate gains in hypertrophy. It should be noted that a decrease in hypertrophy has traditionally been associated with age. In the present meta-analysis, it has been shown that, regardless of the technique used to estimate muscle hypertrophy, age does not have a moderating component on it. These results are as expected, as the included studies focused only on participants between 18 and 40 years old. In humans, sarcopenia (i.e., loss of muscle mass and function) affects individuals from approximately the 4th decade of life [134], with a decrease of 30-50\% in skeletal muscle mass and function by the time individuals reach approximately 80 years of age [135]. For instance, Morton et al.'s [6] study found that RT in addition of protein supplementation is more effective at improving FFM in young or resistance-trained individuals than in older or untrained individuals. It is clear that older individuals are anabolically resistant [136]; however, according to the current meta-analysis, it seems that RT induces similar gains in muscle mass, independently of the age of the participant, when considering individuals from 18 to 40 years old. In any case, future studies are needed in which it is analyzed whether the gains of muscle mass after RT are dependent on the age of the participants of said programmes, including in those studies that have a sample with a wide age range.

Furthermore, in relation to the initial level of the participants (training status) we observed the shorter the experience in RT of the athlete is, the higher the hypertrophy gains are, except for those with an experience of 4 years or more (Figure 4). We believe that the reasons for the similar improvements in sedentary and advanced subjects are due to different reasons. In the case of sedentary or lightly trained 
it is probably the lack of previous stimulus that causes greater hypertrophy. These results are in line with different studies that have shown that hypertrophic responses to RT have been shown to diminish over time [137], in addition to hypertrophic potential being lower in well-trained strength athletes [138]. However, regarding the most advanced practitioners our results are quite surprising. It seems that once an experience threshold of approximately 4 years is surpassed the ability to hypertrophy increase. We hypothesise that this might be due to a more advance knowledge about training methodologies, higher level of adherence to the RT sessions, greater motivation toward training [139], a better exercises technique or even or the use of undeclared anabolic steroids. However, more studies elucidating the factors (behavioural, physiological or other) responsible for this are needed. Finally, from these results we can recommend that if we want high gains in hypertrophy with intermediate trained participants, we will have to be very precise in the design and establishment of the appropriate training load, as well as in the nutritional strategies necessary for an optimal anabolic balance of athletes [6]. Therefore, the previous state of training is a decisive variable in the potential gain of muscle mass. If analysed as a dichotomous variable (trained/untrained, Figure 3), the result is different to that of making a more detailed analysis taking into account several categories of experience (Figure 4). In any case, the relationship between hypertrophy and the training status does not seem linear. It should also be remembered that one possible explanation for this is that there are not enough studies with intermediate training states. Finally, only men were included in the present study because the ability to hypertrophy is very different depending on the sex [10]. In addition, several studies suggest that the menstrual cycle can influence muscle mass improvements [140,141].

\subsection{Training Characteristics}

With respect to the training characteristics the only variable that seems to have a significant moderating effect is the number of series per session, being its moderator coefficient negative $(-0.03$; $p=0.04)$. This suggests that a high number of series per session could negatively affect muscle mass gains. This finding is in line with those by Schoenfeld et al. $[130,132]$ showing that when equaling volume, greater hypertrophy was observed in those groups that distributed the sets with a higher weekly frequency (2-3 days) per muscle group. Contrary to these results (ours and previous) there is a meta-analysis supporting the efficacy of greater exercise volume [142], concluding that individuals interested in achieving maximal hypertrophy should perform a minimum of 2-3 sets per exercise, and that possibly 4-6 sets could give even a greater response; yet still, considerable heterogeneity was present in the analysis [143], and the only significant difference was observed when comparing one set with three sets. Nevertheless, even if a minimum number of series seems to be necessary to maximise muscle hypertrophy [144] there also appears to be a threshold by which the increase in the sets of exercise performed per muscle group within a given training session does not necessarily lead to greater muscle growth $[144,145]$. Thus, when designing a RT programme aiming to increase muscle mass it is not recommended to include an excessively high number of sets, such as that found in this study (16 sets per session on average). Similar recommendations have recently been proposed in a narrative review suggesting that despite increasing the number sets per exercise (albeit the majority of studies within RT literature focus on number of sets), it is likely more beneficial to increase the training frequency [145].

Therefore, future analyses are necessary in which all the variables related to the training load are stratified so that they can give us an idea of which is the optimal dose. Consequently, although we observed an influence of number of sets over the quantity of muscle hypertrophy developed, more studies are needed.

However, perhaps the methods used so far for the muscle hypertrophy may not be sensitive enough to predict which variable and to what extent said variable can moderate greater gains in the development of hypertrophy. 


\section{Conclusions}

In conclusion, resistance training has a significant effect on the improvement of hypertrophy, regardless of the method used to quantify it. The improvements ranged from $1.6 \mathrm{~kg}$ in FFM, $1.7 \mathrm{~kg}$ in LMM, to $1.1 \mathrm{~kg}$ in MM. Regarding the characteristics of the participants, there are no variables (neither the age, nor the training status of the participants) that moderate the gains in hypertrophy. In addition, with respect to the characteristics of the training, the only single variable that moderates inversely the gains in hypertrophy is the number of sets per workout, showing that an excess of sets per workouts affects negatively the amount of muscle growth.

\section{Limitations}

As mentioned earlier, RT has a long and significant effect on the development of hypertrophy. However, these gains were different depending on the variable used as the equivalent of muscle hypertrophy. Clearly, the methodologies used differ from each other, finding a non-significant difference of $45.6-54.6 \%$ when comparing hypertrophy by means of FFM and LMM versus MM. In the context of body composition and muscle hypertrophy, it is important to be very clear about what these two concepts mean and how they are measured as variables. Ideally, muscle mass should be measured isolated from the rest of the tissues, but this can only be achieved by using nuclear magnetic resonance and most of the studies do not utilize this method. Therefore, very few studies assess muscle mass as an isolated variable from the rest of the tissues and/or provide the value for the whole-body muscle mass $[13,15,146]$.

The methodology of assessment could be a limitation, given that the measurement techniques (i.e., DXA, bioimpedance, etc.) of each study are different. However, previous studies have reported that these differences are not significant [147], supporting the comparison made in the present work.

In addition, we need to keep in mind that many of the included studies did not specifically aimed to increase muscle mass, and therefore the heterogeneity of the results may be due to the different methodologies employed. Nevertheless, the purpose of our study was to define the range of muscle mass growths that can be expected after a RT intervention, even if the main purpose of the programmes was not muscle hypertrophy.

Finally, the lack of heterogeneity in the variance of the studies include in the present meta-analysis, the different purposes and outcomes expected by the studies included, and poor description of training variables may be limiting to the present meta-analysis and meta-regression.

Supplementary Materials: The following are available online at http://www.mdpi.com/1660-4601/17/4/1285/s1, Table S1: Main characteristics of the included studies in the present review.

Author Contributions: P.J.B. and D.J.R.-C. carried out the screenings and reviews. J.Á.R.-A. and R.C. carried out the analysis of the articles. P.E.A. and P.J.B. drafted and revised the manuscript. P.J.B., R.C., D.J.R.-C., P.E.A. and J.Á.R.-A. revised the manuscript. All authors have read and agreed to the published version of the manuscript.

Funding: This research received no external funding.

Acknowledgments: In this section you can acknowledge any support given which is not covered by the author contribution or funding sections. This may include administrative and technical support, or donations in kind (e.g., materials used for experiments).

Conflicts of Interest: The authors declare no conflict of interest.

\section{References}

1. Argilés, J.M.; Campos, N.; Lopez-Pedrosa, J.M.; Rueda, R.; Rodriguez-Mañas, L. Skeletal Muscle Regulates Metabolism via Interorgan Crosstalk: Roles in Health and Disease. J. Am. Med. Dir. Assoc. 2016, 17, 789-796. [CrossRef]

2. Abe, T.; Nahar, V.K.; Young, K.C.; Patterson, K.M.; Stover, C.D.; Lajza, D.G.; Tribby, A.C.; Geddam, D.A.; Ford, M.A.; Bass, M.A.; et al. Skeletal muscle mass, bone mineral density, and walking performance in masters cyclists. Rejuvenation Res. 2014, 17, 291-296. [CrossRef] 
3. Schoenfeld, B. Science and Development of Muscle Hypertrophy; Human Kinetics: Pudsey, UK, 2016.

4. Hall, K.D. Predicting metabolic adaptation, body weight change, and energy intake in humans. Am. J. Physiol. Endocrinol. Metab. 2010, 298, E449-E466. [CrossRef]

5. Hall, K.D.; Sacks, G.; Chandramohan, D.; Chow, C.C.; Wang, Y.C.; Gortmaker, S.L.; Swinburn, B.A. Quantification of the effect of energy imbalance on bodyweight. Lancet 2011, 378, 826-837. [CrossRef]

6. Morton, R.W.; Murphy, K.T.; McKellar, S.R.; Schoenfeld, B.J.; Henselmans, M.; Helms, E.; Aragon, A.A.; Devries, M.C.; Banfield, L.; Krieger, J.W.; et al. A systematic review, meta-analysis and meta-regression of the effect of protein supplementation on resistance training-induced gains in muscle mass and strength in healthy adults. Br. J. Sports Med. 2018, 52, 376-384. [CrossRef] [PubMed]

7. Torres, M.; Trexler, E.T.; Smith-Ryan, A.E.; Reynolds, A. A mathematical model of the effects of resistance exercise-induced muscle hypertrophy on body composition. Eur. J. Appl. Physiol. 2018, 118, 449-460. [CrossRef] [PubMed]

8. Mangine, G.T.; Hoffman, J.R.; Gonzalez, A.M.; Townsend, J.R.; Wells, A.J.; Jajtner, A.R.; Beyer, K.S.; Boone, C.H.; Miramonti, A.A.; Wang, R.; et al. The effect of training volume and intensity on improvements in muscular strength and size in resistance-trained men. Physiol. Rep. 2015, 3, e12472. [CrossRef]

9. Nybo, L.; Sundstrup, E.; Jakobsen, M.D.; Mohr, M.; Hornstrup, T.; Simonsen, L.; Bulow, J.; Randers, M.B.; Nielsen, J.J.; Aagaard, P.; et al. High-Intensity Training versus Traditional Exercise Interventions for Promoting Health. Med. Sci. Sports Exerc. 2010, 42, 1951-1958. [CrossRef]

10. Ahtiainen, J.P.; Walker, S.; Peltonen, H.; Holviala, J.; Sillanpaa, E.; Karavirta, L.; Sallinen, J.; Mikkola, J.; Valkeinen, H.; Mero, A.; et al. Heterogeneity in resistance training-induced muscle strength and mass responses in men and women of different ages. Age 2016, 38, 10. [CrossRef]

11. Moher, D.; Liberati, A.; Tetzlaff, J.; Altman, D.G.; Group, P. Preferred reporting items for systematic reviews and meta-analyses: The PRISMA statement. Ann. Intern. Med. 2009, 151, 264-269. [CrossRef]

12. Bartolomei, S.; Hoffman, J.R.; Stout, J.R.; Merni, F. Effect of Lower-Body Resistance Training on Upper-Body Strength Adaptation in Trained Men. J. Strength Cond. Res. 2018, 32, 13-18. [CrossRef] [PubMed]

13. Nunes, J.P.; Ribeiro, A.S.; Schoenfeld, B.J.; Tomeleri, C.M.; Avelar, A.; Trindade, M.C.; Nabuco, H.C.; Cavalcante, E.F.; Junior, P.S.; Fernandes, R.R.; et al. Creatine supplementation elicits greater muscle hypertrophy in upper than lower limbs and trunk in resistance-trained men. Nutr. Health 2017, 23, $223-229$. [CrossRef] [PubMed]

14. Heymsfield, S.B.; Smith, R.; Aulet, M.; Bensen, B.; Lichtman, S.; Wang, J.; Pierson, R.N., Jr. Appendicular skeletal muscle mass: Measurement by dual-photon absorptiometry. Am. J. Clin. Nutr. 1990, 52, $214-218$. [CrossRef] [PubMed]

15. Kim, J.; Heshka, S.; Gallagher, D.; Kotler, D.P.; Mayer, L.; Albu, J.; Shen, W.; Freda, P.U.; Heymsfield, S.B. Intermuscular adipose tissue-free skeletal muscle mass: Estimation by dual-energy X-ray absorptiometry in adults. J. Appl. Physiol. 2004, 97, 655-660. [CrossRef] [PubMed]

16. Higgins, J.P.; Thompson, S.G.; Deeks, J.J.; Altman, D.G. Measuring inconsistency in meta-analyses. BMJ 2003, 327, 557-560. [CrossRef] [PubMed]

17. Abe, T.; DeHoyos, D.V.; Pollock, M.L.; Garzarella, L. Time course for strength and muscle thickness changes following upper and lower body resistance training in men and women. Eur. J. Appl. Physiol. 2000, 81, 174-180. [CrossRef] [PubMed]

18. Ahtiainen, J.P.; Hulmi, J.J.; Kraemer, W.J.; Lehti, M.; Nyman, K.; Selanne, H.; Alen, M.; Pakarinen, A.; Komulainen, J.; Kovanen, V.; et al. Heavy resistance exercise training and skeletal muscle androgen receptor expression in younger and older men. Steroids 2011, 76, 183-192. [CrossRef]

19. Alvarez, M.; Sedano, S.; Cuadrado, G.; Carlos Redondo, J. Effects of an 18-week strength training program on low-handicap golfers' performance. J. Strength Cond. Res. 2012, 26, 1110-1121. [CrossRef]

20. Alvehus, M.; Boman, N.; Soderlund, K.; Svensson, M.B.; Buren, J. Metabolic adaptations in skeletal muscle, adipose tissue, and whole-body oxidative capacity in response to resistance training. Eur. J. Appl. Physiol. 2014, 114, 1463-1471. [CrossRef]

21. Arazi, H.; Rohani, H.; Ghiasi, A.; Keikanloo, N.A. Resistance training \& beta-hydroxy-beta-methylbutyrate supplementation on hormones. Rev. Bras. Med. Esporte 2015, 21, 386-389.

22. Arciero, P.J.; Hannibal, N.S., III; Nindl, B.C.; Gentile, C.L.; Hamed, J.; Vukovich, M.D. Comparison of creatine ingestion and resistance training on energy expenditure and limb blood flow. Metabolism 2001, 50, 1429-1434. [CrossRef] 
23. Bang, H.S.; Seo, D.Y.; Chung, Y.M.; Kim, D.H.; Lee, S.J.; Lee, S.R.; Kwak, H.B.; Kim, T.N.; Kim, M.; Oh, K.M.; et al. Ursolic acid supplementation decreases markers of skeletal muscle damage during resistance training in resistance-trained men: A pilot study. Korean J. Physiol. Pharmacol. 2017, 21, 651-656. [CrossRef] [PubMed]

24. Bartolomei, S.; Hoffman, J.R.; Stout, J.R.; Zini, M.; Stefanelli, C.; Merni, F. Comparison of block versus weekly undulating periodization models on endocrine and strength changes in male athletes. Kinesiology 2016, 48, 71-78. [CrossRef]

25. Bhasin, S.; Storer, T.W.; Berman, N.; Callegari, C.; Clevenger, B.; Phillips, J.; Bunnell, T.J.; Tricker, R.; Shirazi, A.; Casaburi, R. The effects of supraphysiologic doses of testosterone on muscle size and strength in normal men. N. Engl. J. Med. 1996, 335, 1-7. [CrossRef] [PubMed]

26. Buresh, R.; Berg, K.; French, J. The effect of resistive exercise rest interval on hormonal response, strength, and hypertrophy with training. J. Strength Cond. Res. 2009, 23, 62-71. [CrossRef] [PubMed]

27. Chromiak, J.A.; Smedley, B.; Carpenter, W.; Brown, R.; Koh, Y.S.; Lamberth, J.G.; Joe, L.A.; Abadie, B.R.; Altorfer, G. Effect of a 10-week strength training program and recovery drink on body composition, muscular strength and endurance, and anaerobic power and capacity. Nutrition 2004, 20, 420-427. [CrossRef] [PubMed]

28. Colquhoun, R.J.; Gai, C.M.; Walters, J.; Brannon, A.R.; Kilpatrick, M.W.; D’Agostino, D.P.; Campbell, W.I. Comparison of powerlifting performance in trained men using traditional and flexible daily undulating periodization. J. Strength Cond. Res. 2017, 31, 283-291. [CrossRef]

29. Crewther, B.T.; Heke, T.; Keogh, J.W.L. The effects of a resistance-training program on strength, body composition and baseline hormones in male athletes training concurrently for rugby union 7's. J. Sports Med. Phys. Fit. 2013, 53, 34-41.

30. Deruisseau, K.C.; Roberts, L.M.; Kushnick, M.R.; Evans, A.M.; Austin, K.; Haymes, E.M. Iron status of young males and females performing weight-training exercise. Med. Sci. Sports Exerc. 2004, 36, 241-248. [CrossRef]

31. Fu, Y.; Karsten, B.; Larumbe-Zabala, E.; Seijo, M.; Naclerio, F. Comparison of Two Equated Resistance Training Weekly Volume Routines Using Different Frequencies on Body Composition and Performance in Trained Males. Appl. Physiol. Nutr. Metab. 2017, 43, 475-481.

32. Gallagher, P.M.; Carrithers, J.A.; Godard, M.P.; Schulze, K.E.; Trappe, S.W. beta-hydroxy-beta-methylbutyrate ingestion, Part I: Effects on strength and fat free mass. Med. Sci. Sports Exerc. 2000, 32, 2109-2115. [CrossRef] [PubMed]

33. Garthe, I.; Raastad, T.; Refsnes, P.E.; Sundgot-Borgen, J. Effect of nutritional intervention on body composition and performance in elite athletes. Eur. J. Sport Sci. 2013, 13, 295-303. [CrossRef] [PubMed]

34. Ghahramanloo, E.; Midgley, A.W.; Bentley, D.J. The Effect of Concurrent Training on Blood Lipid Profile and Anthropometrical Characteristics of Previously Untrained Men. J. Phys. Act. Health 2009, 6, 760-766. [CrossRef]

35. Hong, A.R.; Hong, S.M.; Shin, Y.A. Effects of Resistance Training on Muscle Strength, Endurance, and Motor Unit According to Ciliary Neurotrophic Factor Polymorphism in Male College Students. J. Sports Sci. Med. 2014, 13, 680-688. [PubMed]

36. Hu, M.; Finni, T.; Zou, L.; Perhonen, M.; Sedliak, M.; Alen, M.; Cheng, S. Effects of strength training on work capacity and parasympathetic heart rate modulation during exercise in physically inactive men. Int. J. Sports Med. 2009, 30, 719-724. [CrossRef] [PubMed]

37. Huso, M.E.; Hampl, J.S.; Johnston, C.S.; Swan, P.D. Creatine supplementation influences substrate utilization at rest. J. Appl. Physiol. 2002, 93, 2018-2022. [CrossRef] [PubMed]

38. Jang, J.; Park, H.Y.; Yoo, C.; Park, Y.; Kim, J.; Lim, K. The synergistic effect of protein complex supplementation combined with 12 weeks of resistance training on isokinetic muscular function in untrained young males. $J$. Exerc. Nutr. Biochem. 2017, 21, 27-36. [CrossRef]

39. Joy, J.M.; Gundermann, D.M.; Lowery, R.P.; Jaeger, R.; McCleary, S.A.; Purpura, M.; Roberts, M.D.; Wilson, S.M.C.; Hornberger, T.A.; Wilson, J.M. Phosphatidic acid enhances mTOR signaling and resistance exercise induced hypertrophy. Nutr. Metab. 2014, 11, 29. [CrossRef]

40. Kim, H.H.; Kim, Y.J.; Lee, S.Y.; Jeong, D.W.; Lee, J.G.; Yi, Y.H.; Cho, Y.H.; Choi, E.J.; Kim, H.J. Interactive effects of an isocaloric high-protein diet and resistance exercise on body composition, ghrelin, and metabolic and hormonal parameters in untrained young men: A randomized clinical trial. J. Diabetes Investig. 2014, 5 , 242-247. [CrossRef] 
41. Kon, M.; Ohiwa, N.; Honda, A.; Matsubayashi, T.; Ikeda, T.; Akimoto, T.; Suzuki, Y.; Hirano, Y.; Russell, A.P. Effects of systemic hypoxia on human muscular adaptations to resistance exercise training. Physiol. Rep. 2014, 2, e12033. [CrossRef] [PubMed]

42. Kraemer, W.J.; Hatfield, D.L.; Volek, J.S.; Fragala, M.S.; Vingren, J.L.; Anderson, J.M.; Spiering, B.A.; Thomas, G.A.; Ho, J.Y.; Quann, E.E.; et al. Effects of amino acids supplement on physiological adaptations to resistance training. Med. Sci. Sports Exerc. 2009, 41, 1111-1121. [CrossRef]

43. Kreipke, V.C.; Allman, B.R.; Kinsey, A.W.; Moffatt, R.J.; Hickner, R.C.; Ormsbee, M.J. Impact of four weeks of a multi-ingredient performance supplement on muscular strength, body composition, and anabolic hormones in resistance-trained young men. J. Strength Cond. Res. 2015, 29, 3453-3465. [CrossRef] [PubMed]

44. Lemmer, J.T.; Ivey, F.M.; Ryan, A.S.; Martel, G.F.; Hurlbut, D.E.; Metter, J.E.; Fozard, J.L.; Fleg, J.L.; Hurley, B.F. Effect of strength training on resting metabolic rate and physical activity: Age and gender comparisons. Med. Sci. Sports Exerc. 2001, 33, 532-541. [CrossRef] [PubMed]

45. Li, X.; Wang, S.J.; Tan, S.C.; Chew, P.L.; Liu, L.; Wang, L.; Wen, L.; Ma, L. The A55T and K153R polymorphisms of MSTN gene are associated with the strength training-induced muscle hypertrophy among Han Chinese men. J. Sports Sci. 2014, 32, 883-891. [CrossRef] [PubMed]

46. Lockwood, C.M.; Roberts, M.D.; Dalbo, V.J.; Smith-Ryan, A.E.; Kendall, K.L.; Moon, J.R.; Stout, J.R. Effects of Hydrolyzed Whey versus Other Whey Protein Supplements on the Physiological Response to 8 Weeks of Resistance Exercise in College-Aged Males. J. Am. Coll. Nutr. 2017, 36, 16-27. [CrossRef] [PubMed]

47. Lukaski, H.C.; Bolonchuk, W.W.; Siders, W.A.; Milne, D.B. Chromium supplementation and resistance training: Effects on body composition, strength, and trace element status of men. Am. J. Clin. Nutr. 1996, 63, 954-965. [CrossRef] [PubMed]

48. Mazzetti, S.A.; Kraemer, W.J.; Volek, J.S.; Duncan, N.D.; Ratamess, N.A.; Gomez, A.L.; Newton, R.U.; Hakkinen, K.; Fleck, S.J. The influence of direct supervision of resistance training on strength performance. Med. Sci. Sports Exerc. 2000, 32, 1175-1184. [CrossRef] [PubMed]

49. McCartney, N.; Hicks, A.L.; Martin, J.; Webber, C.E. Long-term resistance training in the elderly: Effects on dynamic strength, exercise capacity, muscle, and bone. J. Gerontol. Ser. A Biol. Sci. Med. Sci. 1995, 50, B97-B104. [CrossRef]

50. Naclerio, F.; Larumbe-Zabala, E.; Ashrafi, N.; Seijo, M.; Nielsen, B.; Allgrove, J.; Earnest, C.P. Effects of protein-carbohydrate supplementation on immunity and resistance training outcomes: A double-blind, randomized, controlled clinical trial. Eur. J. Appl. Physiol. 2017, 117, 267-277. [CrossRef] [PubMed]

51. Noonan, D.; Berg, K.; Latin, R.W.; Wagner, J.C.; Reimers, K. Effects of varying dosages of oral creatine relative to fat free body mass on strength and body composition. J. Strength Cond. Res. 1998, 12, 104-108.

52. Oliver, J.M.; Jagim, A.R.; Sanchez, A.C.; Mardock, M.A.; Kelly, K.A.; Meredith, H.J.; Smith, G.L.; Greenwood, M.; Parker, J.L.; Riechman, S.E.; et al. Greater gains in strength and power with intraset rest intervals in hypertrophic training. J. Strength Cond. Res. 2013, 27, 3116-3131. [CrossRef] [PubMed]

53. Ormsbee, M.J.; Mandler, W.K.; Thomas, D.D.; Ward, E.G.; Kinsey, A.W.; Simonavice, E.; Panton, L.B.; Kim, J.S. The effects of six weeks of supplementation with multi-ingredient performance supplements and resistance training on anabolic hormones, body composition, strength, and power in resistance-trained men. J. Int. Soc. Sports Nutr. 2012, 9, 49. [CrossRef] [PubMed]

54. Ormsbee, M.J.; Thomas, D.D.; Mandler, W.K.; Ward, E.G.; Kinsey, A.W.; Panton, L.B.; Scheett, T.P.; Hooshmand, S.; Simonavice, E.; Kim, J.S. The effects of pre- and post-exercise consumption of multi-ingredient performance supplements on cardiovascular health and body fat in trained men after six weeks of resistance training: A stratified, randomized, double-blind study. Nutr. Metab. 2013, 10, 39. [CrossRef] [PubMed]

55. Piirainen, J.M.; Salmi, J.A.; Avela, J.; Linnamo, V. Effect of body composition on the neuromuscular function of finnish conscripts during an 8-week basic training period. J. Strength Cond. Res. 2008, 22, 1916-1925. [CrossRef] [PubMed]

56. Perez-Gomez, J.; Vicente-Rodriguez, G.; Ara Royo, I.; Martinez-Redondo, D.; Puzo Foncillas, J.; Moreno, L.A.; Diez-Sanchez, C.; Casajus, J.A. Effect of endurance and resistance training on regional fat mass and lipid profile. Nutr. Hosp. 2013, 28, 340-346.

57. Radaelli, R.; Fleck, S.J.; Leite, T.; Leite, R.D.; Pinto, R.S.; Fernandes, L.; Simao, R. Dose-response of 1, 3, and 5 sets of resistance exercise on strength, local muscular endurance, and hypertrophy. J. Strength Cond. Res. 2015, 29, 1349-1358. [CrossRef] 
58. Ribeiro, A.S.; Schoenfeld, B.J.; Silva, D.R.; Pina, F.L.; Guariglia, D.A.; Porto, M.; Maesta, N.; Burini, R.C.; Cyrino, E.S. Effect of Two-Versus Three-Way Split Resistance Training Routines on Body Composition and Muscular Strength in Bodybuilders: A Pilot Study. Int. J. Sport Nutr. Exerc. Metab. 2015, 25, 559-565. [CrossRef]

59. Roberts, M.D.; Iosia, M.; Kerksick, C.M.; Taylor, L.W.; Campbell, B.; Wilborn, C.D.; Harvey, T.; Cooke, M.; Rasmussen, C.; Greenwood, M.; et al. Effects of arachidonic acid supplementation on training adaptations in resistance-trained males. J. Int. Soc. Sports Nutr. 2007, 4, 21. [CrossRef]

60. Rogerson, S.; Riches, C.J.; Jennings, C.; Weatherby, R.P.; Meir, R.A.; Marshall-Gradisnik, S.M. The effect of five weeks of Tribulus terrestris supplementation on muscle strength and body composition during preseason training in elite rugby league players. J. Strength Cond. Res. 2007, 21, 348-353.

61. Schumann, M.; Kuusmaa, M.; Newton, R.U.; Sirparanta, A.I.; Syvaoja, H.; Hakkinen, A.; Hakkinen, K. Fitness and lean mass increases during combined training independent of loading order. Med. Sci. Sports Exerc. 2014, 46, 1758-1768. [CrossRef]

62. Shelmadine, B.; Cooke, M.; Buford, T.; Hudson, G.; Redd, L.; Leutholtz, B.; Willoughby, D.S. Effects of 28 days of resistance exercise and consuming a commercially available pre-workout supplement, NO-Shotgun(R), on body composition, muscle strength and mass, markers of satellite cell activation, and clinical safety markers in males. J. Int. Soc. Sports Nutr. 2009, 6, 16. [CrossRef] [PubMed]

63. Snijders, T.; Res, P.T.; Smeets, J.S.; van Vliet, S.; van Kranenburg, J.; Maase, K.; Kies, A.K.; Verdijk, L.B.; van Loon, L.J. Protein Ingestion before Sleep Increases Muscle Mass and Strength Gains during Prolonged Resistance-Type Exercise Training in Healthy Young Men. J. Nutr. 2015, 145, 1178-1184. [CrossRef] [PubMed]

64. Spence, A.L.; Naylor, L.H.; Carter, H.H.; Buck, C.L.; Dembo, L.; Murray, C.P.; Watson, P.; Oxborough, D.; George, K.P.; Green, D.J. A prospective randomised longitudinal MRI study of left ventricular adaptation to endurance and resistance exercise training in humans. J. Physiol. 2011, 589, 5443-5452. [CrossRef] [PubMed]

65. Spillane, M.; Schoch, R.; Cooke, M.; Harvey, T.; Greenwood, M.; Kreider, R.; Willoughby, D.S. The effects of creatine ethyl ester supplementation combined with heavy resistance training on body composition, muscle performance, and serum and muscle creatine levels. J. Int. Soc. Sports Nutr. 2009, 6, 6. [CrossRef]

66. Spillane, M.; Schwarz, N.; Leddy, S.; Correa, T.; Minter, M.; Longoria, V.; Willoughby, D.S. Effects of 28 days of resistance exercise while consuming commercially available pre- and post-workout supplements, NO-Shotgun (R) and NO-Synthesize (R) on body composition, muscle strength and mass, markers of protein synthesis, and clinical safety markers in males. Nutr. Metab. 2011, 8, 78.

67. Terzis, G.; Georgiadis, G.; Stratakos, G.; Vogiatzis, I.; Kavouras, S.; Manta, P.; Mascher, H.; Blomstrand, E. Resistance exercise-induced increase in muscle mass correlates with p70S6 kinase phosphorylation in human subjects. Eur. J. Appl. Physiol. 2008, 102, 145-152. [CrossRef]

68. Terzis, G.; Stratakos, G.; Manta, P.; Georgiadis, G. Throwing performance after resistance training and detraining. J. Strength Cond. Res. 2008, 22, 1198-1204. [CrossRef]

69. Thomson, J.S.; Watson, P.E.; Rowlands, D.S. Effects of nine weeks of beta-HYDROXYbeta-METHYLBUTYRATE supplementation on strength and body composition in resistance trained men. J. Strength Cond. Res. 2009, 23, 827-835. [CrossRef]

70. Tomljanovic, M.; Spasic, M.; Gabrilo, G.; Uljevic, O.; Foretic, N. Effects of five weeks of functional vs. Traditional resistance training on anthropometric and motor performance variables. Kinesiology 2011, 43, 145-154.

71. Wilborn, C.; Taylor, L.; Poole, C.; Foster, C.; Willoughby, D.; Kreider, R. Effects of a Purported Aromatase and 5 alpha-Reductase Inhibitor on Hormone Profiles in College-Age Men. Int. J. Sport Nutr. Exerc. Metab. 2010, 20, 457-465. [CrossRef]

72. Willoughby, D.S.; Spillane, M.; Schwarz, N. Heavy Resistance Training and Supplementation with the Alleged Testosterone Booster NMDA Has No Effect on Body Composition, Muscle Performance, and Serum Hormones Associated with the Hypothalamo-Pituitary-Gonadal Axis in Resistance-Trained Males. J. Sports Sci. Med. 2014, 13, 192-199. [PubMed]

73. Willoughby, D.S.; Stout, J.R.; Wilborn, C.D. Effects of resistance training and protein plus amino acid supplementation on muscle anabolism, mass, and strength. Amino Acids 2007, 32, 467-477. [CrossRef] [PubMed] 
74. Wilson, J.M.; Joy, J.M.; Lowery, R.P.; Roberts, M.D.; Lockwood, C.M.; Manninen, A.H.; Fuller, J.C., Jr.; De Souza, E.O.; Baier, S.M.; Wilson, S.M.C.; et al. Effects of oral adenosine-5'-triphosphate supplementation on athletic performance, skeletal muscle hypertrophy and recovery in resistance-trained men. Nutr. Metab. 2013, 10, 57. [CrossRef] [PubMed]

75. Wilson, J.M.; Lowery, R.P.; Joy, J.M.; Andersen, J.C.; Wilson, S.M.; Stout, J.R.; Duncan, N.; Fuller, J.C.; Baier, S.M.; Naimo, M.A.; et al. The effects of 12 weeks of beta-hydroxy-beta-methylbutyrate free acid supplementation on muscle mass, strength, and power in resistance-trained individuals: A randomized, double-blind, placebo-controlled study. Eur. J. Appl. Physiol. 2014, 114, 1217-1227. [CrossRef]

76. Zamani, M.; Peeri, M. The Effect of Body Part-dependent Resistance Training on Lipid Profiles and Hormonal Levels in Young Males. Ann. Appl. Sport Sci. 2017, 5, 51-58. [CrossRef]

77. Alcaraz, P.E.; Perez-Gomez, J.; Chavarrias, M.; Blazevich, A.J. Similarity in adaptations to high-resistance circuit vs. traditional strength training in resistance-trained men. J. Strength Cond. Res. 2011, 25, 2519-2527. [CrossRef]

78. Andre, T.L.; Gann, J.J.; McKinley-Barnard, S.K.; Song, J.J.; Willoughby, D.S. Eight Weeks of Phosphatidic Acid Supplementation in Conjunction with Resistance Training Does Not Differentially Affect Body Composition and Muscle Strength in Resistance-Trained Men. J. Sports Sci. Med. 2016, 15, 532-539.

79. Ara, I.; Perez-Gomez, J.; Vicente-Rodriguez, G.; Chavarren, J.; Dorado, C.; Calbet, J.A.L. Serum free testosterone, leptin and soluble leptin receptor changes in a 6-week strength-training programme. Br. J. Nutr. 2007, 96, 1053-1059. [CrossRef]

80. Bemben, M.G.; Bemben, D.A.; Loftiss, D.D.; Knehans, A.W. Creatine supplementation during resistance training in college football athletes. Med. Sci. Sports Exerc. 2001, 33, 1667-1673. [CrossRef]

81. Brown, G.A.; Vukovich, M.D.; Sharp, R.L.; Reifenrath, T.A.; Parsons, K.A.; King, D.S. Effect of oral DHEA on serum testosterone and adaptations to resistance training in young men. J. Appl. Physiol. 1999, 87, 2274-2283. [CrossRef]

82. Burke, D.G.; Chilibeck, P.D.; Davison, K.S.; Candow, D.G.; Farthing, J.; Smith-Palmer, T. The effect of whey protein supplementation with and without creatine monohydrate combined with resistance training on lean tissue mass and muscle strength. Int. J. Sport Nutr. Exerc. Metab. 2001, 11, 349-364. [CrossRef] [PubMed]

83. Caldow, M.K.; Thomas, E.E.; Dale, M.J.; Tomkinson, G.R.; Buckley, J.D.; Cameron-Smith, D. Early myogenic responses to acute exercise before and after resistance training in young men. Physiol. Rep. 2015, 3, e12511. [CrossRef] [PubMed]

84. Chycki, J.; Czuba, M.; Golas, A.; Zajac, A.; Fidos-Czuba, O.; Mlynarz, A.; Smolka, W. Neuroendocrine Responses and Body Composition Changes Following Resistance Training Under Normobaric Hypoxia. J. Hum. Kinet. 2016, 53, 91-98. [CrossRef] [PubMed]

85. Deyssig, R.; Frisch, H.; Blum, W.F.; Waldhor, T. Effect of growth hormone treatment on hormonal parameters, body composition and strength in athletes. Eur. J. Endocrinol. 1993, 128, 313-318. [CrossRef] [PubMed]

86. Escalante, G.; Alencar, M.; Haddock, B.; Harvey, P. The effects of phosphatidic acid supplementation on strength, body composition, muscular endurance, power, agility, and vertical jump in resistance trained men. J. Int. Soc. Sports Nutr. 2016, 13, 24. [CrossRef]

87. Fahey, T.D.; Brown, C.H. Effects of an anabolic steroid on strength, body composition, and endurance of college males when accompanied by a weight training-program. Med. Sci. Sports Exerc. 1973, 5, 272-276. [CrossRef]

88. Falk, D.J.; Heelan, K.A.; Thyfault, J.P.; Koch, A.J. Effects of effervescent creatine, ribose, and glutamine supplementation on muscular strength, muscular endurance, and body composition. J. Strength Cond. Res. 2003, 17, 810-816.

89. Fyfe, J.J.; Bartlett, J.D.; Hanson, E.D.; Stepto, N.K.; Bishop, D.J. Endurance Training Intensity Does Not Mediate Interference to Maximal Lower-Body Strength Gain during Short-Term Concurrent Training. Front. Physiol. 2016, 7, 487. [CrossRef]

90. Glowacki, S.P.; Martin, S.E.; Maurer, A.N.N.; Baek, W.; Green, J.S.; Crouse, S.F. Effects of Resistance, Endurance, and Concurrent Exercise on Training Outcomes in Men. Med. Sci. Sports Exerc. 2004, 36, 2119-2127. [CrossRef] 
91. Gobbo, L.A.; Ritti-Dias, R.M.; Avelar, A.; Silva, A.M.; Coelho-e-Silva, M.J.; Cyrino, E.S. Changes in skeletal muscle mass assessed by anthropometric equations after resistance training. Int. J. Sports Med. 2013, 34, 28-33. [CrossRef]

92. Harber, M.P.; Fry, A.C.; Rubin, M.R.; Smith, J.C.; Weiss, L.W. Skeletal muscle and hormonal adaptations to circuit weight training in untrained men. Scand. J. Med. Sci. Sports 2004, 14, 176-185. [CrossRef]

93. Hoffman, J.R.; Ratamess, N.A.; Tranchina, C.P.; Rashti, S.L.; Kang, J.; Faigenbaum, A.D. Effect of Protein-Supplement Timing on Strength, Power, and Body-Composition Changes in Resistance-Trained Men. Int. J. Sport Nutr. Exerc. Metab. 2009, 19, 172-185. [CrossRef] [PubMed]

94. Hoffman, J.R.; Stout, J.R.; Williams, D.R.; Wells, A.J.; Fragala, M.S.; Mangine, G.T.; Gonzalez, A.M.; Emerson, N.S.; McCormack, W.P.; Scanlon, T.C.; et al. Efficacy of phosphatidic acid ingestion on lean body mass, muscle thickness and strength gains in resistance-trained men. J. Int. Soc. Sports Nutr. 2012, 9, 47. [CrossRef] [PubMed]

95. Ihalainen, J.K.; Peltonen, H.; Paulsen, G.; Ahtiainen, J.P.; Taipale, R.S.; Hamalainen, M.; Moilanen, E.; Mero, A.A. Inflammation status of healthy young men: Initial and specific responses to resistance training. Appl. Physiol. Nutr. Metab. 2018, 43, 252-258. [CrossRef] [PubMed]

96. Joy, J.M.; Vogel, R.M.; Moon, J.R.; Falcone, P.H.; Mosman, M.M.; Kim, M.P. Twelve weeks supplementation with an extended-release caffeine and ATP-enhancing supplement may improve body composition without affecting hematology in resistance-trained men. J. Int. Soc. Sports Nutr. 2016, 13, 25. [CrossRef] [PubMed]

97. Kelly, V.G.; Jenkins, D.G. Effect of oral creatine supplementation on near-maximal strength and repeated sets of high-intensity bench press exercise. J. Strength Cond. Res. 1998, 12, 109-115.

98. Kerksick, C.M.; Wilborn, C.D.; Campbell, W.I.; Harvey, T.M.; Marcello, B.M.; Roberts, M.D.; Parker, A.G.; Byars, A.G.; Greenwood, L.D.; Almada, A.L.; et al. The effects of creatine monohydrate supplementation with and without D-Pinitol on resistance training adaptations. J. Strength Cond. Res. 2009, 23, 2673-2682. [CrossRef] [PubMed]

99. Kerksick, C.M.; Rasmussen, C.J.; Lancaster, S.L.; Magu, B.; Smith, P.; Melton, C.; Greenwood, M.; Almada, A.L.; Earnest, C.P.; Kreider, R.B. The effects of protein and amino acid supplementation on performance and training adaptations during ten weeks of resistance training. J. Strength Cond. Res. 2006, 20, 643-653.

100. King, D.S.; Sharp, R.L.; Vukovich, M.D.; Brown, G.A.; Reifenrath, T.A.; Uhl, N.L.; Parsons, K.A. Effect of oral androstenedione on serum testosterone and adaptations to resistance training in young men: A randomized controlled trial. JAMA 1999, 281, 2020-2028. [CrossRef]

101. Ko, I.G.; Choi, P.B. Regular exercise modulates obesity factors and body composition in sturdy men. J. Exerc. Rehabil. 2013, 9, 256-262. [CrossRef]

102. Kreider, R.B.; Ferreira, M.P.; Greenwood, M.; Wilson, M.; Almada, A.L. Effects of conjugated linoleic acid supplementation during resistance training on body composition, bone density, strength, and selected hematological markers. J. Strength Cond. Res. 2002, 16, 325-334. [PubMed]

103. Lemon, P.W.; Tarnopolsky, M.A.; MacDougall, J.D.; Atkinson, S.A. Protein requirements and muscle mass/strength changes during intensive training in novice bodybuilders. J. Appl. Physiol. 1992, 73, 767-775. [CrossRef] [PubMed]

104. Lo, M.S.; Lin, L.L.C.; Yao, W.J.; Ma, M.C. Training and detraining effects of the Resistance vs Endurance program on body composition, body size, and physical performance in young men. J. Strength Cond. Res. 2011, 25, 2246-2254. [CrossRef] [PubMed]

105. Lowery, R.P.; Joy, J.M.; Loenneke, J.P.; de Souza, E.O.; Machado, M.; Dudeck, J.E.; Wilson, J.M. Practical blood flow restriction training increases muscle hypertrophy during a periodized resistance training programme. Clin. Physiol. Funct. Imaging 2014, 34, 317-321. [CrossRef] [PubMed]

106. Mangine, G.T.; Ratamess, N.A.; Hoffman, J.R.; Faigenbaum, A.D.; Kang, J.; Chilakos, A. The effects of combined ballistic and heavy resistance training on maximal lower- and upper-body strength in recreationally trained men. J. Strength Cond. Res. 2008, 22, 132-139. [CrossRef] [PubMed]

107. Mayhew, D.L.; Kim, J.S.; Cross, J.M.; Ferrando, A.A.; Bamman, M.M. Translational signaling responses preceding resistance training-mediated myofiber hypertrophy in young and old humans. J. Appl. Physiol. 2009, 107, 1655-1662. [CrossRef] [PubMed] 
108. Miller, W.J.; Sherman, W.M.; Ivy, J.L. Effect of strength training on glucose tolerance and post-glucose insulin response. Med. Sci. Sports Exerc. 1984, 16, 539-543. [CrossRef]

109. Moore, D.R.; Del Bel, N.C.; Nizi, K.I.; Hartman, J.W.; Tang, J.E.; Armstrong, D.; Phillips, S.M. Resistance training reduces fasted- and fed-state leucine turnover and increases dietary nitrogen retention in previously untrained young men. J. Nutr. 2007, 137, 985-991. [CrossRef]

110. Paoli, A.; Gentil, P.; Moro, T.; Marcolin, G.; Bianco, A. Resistance Training with Single vs. Multi-joint Exercises at Equal Total Load Volume: Effects on Body Composition, Cardiorespiratory Fitness, and Muscle Strength. Front. Physiol. 2017, 8, 1105. [CrossRef]

111. Peeters, B.M.; Lantz, C.D.; Mayhew, J.L. Effect of oral creatine monohydrate and creatine phosphate supplementation on maximal strength indices, body composition, and blood pressure. J. Strength Cond. Res. 1999, 13, 3-9.

112. Peronnet, F.; Thibault, G.; Perrault, H.; Cousineau, D. Sympathetic response to maximal bicycle exercise before and after leg strength training. Eur. J. Appl. Physiol. Occup. Physiol. 1986, 55, 1-4. [CrossRef]

113. Saremi, A.; Gharakhanloo, R.; Sharghi, S.; Gharaati, M.R.; Larijani, B.; Omidfar, K. Effects of oral creatine and resistance training on serum myostatin and GASP-1. Mol. Cell Endocrinol. 2010, 317, 25-30. [CrossRef] [PubMed]

114. Schneider, S.M.; Amonette, W.E.; Blazine, K.; Bentley, J.; Lee, S.M.; Loehr, J.A.; Moore, A.D., Jr.; Rapley, M.; Mulder, E.R.; Smith, S.M. Training with the International Space Station interim resistive exercise device. Med. Sci. Sports Exerc. 2003, 35, 1935-1945. [CrossRef] [PubMed]

115. Slater, G.; Jenkins, D.; Logan, P.; Lee, H.; Vukovich, M.; Rathmacher, J.A.; Hahn, A.G. beta-hydroxy-beta-methylbutyrate (HMB) supplementation does not affect changes in strength or body composition during resistance training in trained men. Int. J. Sport Nutr. Exerc. Metab. 2001, 11, 384-396. [CrossRef] [PubMed]

116. Spillane, M.; Willoughby, D.S. Daily Overfeeding from Protein and/or Carbohydrate Supplementation for Eight Weeks in Conjunction with Resistance Training Does not Improve Body Composition and Muscle Strength or Increase Markers Indicative of Muscle Protein Synthesis and Myogenesis in Resistance-Trained Males. J. Sports Sci. Med. 2016, 15, 17-25. [PubMed]

117. Taylor, L.; Poole, C.; Pena, E.; Lewing, M.; Kreider, R.; Foster, C.; Wilborn, C. Effects of combined creatine plus fenugreek extract vs. creatine plus carbohydrate supplementation on resistance training adaptations. $J$. Sports Sci. Med. 2011, 10, 254-260. [PubMed]

118. Thorstensson, A.; Hulten, B.; von Dobeln, W.; Karlsson, J. Effect of strength training on enzyme activities and fibre characteristics in human skeletal muscle. Acta Physiol. Scand. 1976, 96, 392-398. [CrossRef]

119. Tinsley, G.M.; Urbina, S.; Mullins, J.; Outlaw, J.; Hayward, S.; Stone, M.; Foster, C.; Wilborn, C.; Taylor, L. Influence of A Thermogenic Dietary Supplement on Safety Markers, Body Composition, Energy Expenditure, Muscular Performance and Hormone Concentrations: A Randomized, Placebo-Controlled, Double-Blind Trial. J. Sports Sci. Med. 2017, 16, 459-467.

120. Volek, J.S.; Ratamess, N.A.; Rubin, M.R.; Gomez, A.L.; French, D.N.; McGuigan, M.M.; Scheett, T.P.; Sharman, M.J.; Hakkinen, K.; Kraemer, W.J. The effects of creatine supplementation on muscular performance and body composition responses to short-term resistance training overreaching. Eur. J. Appl. Physiol. 2004, 91, 628-637. [CrossRef]

121. Yan, B.; Lai, X.; Yi, L.; Wang, Y.; Hu, Y. Effects of five-week resistance training in hypoxia on hormones and muscle strength. J. Strength Cond. Res. 2016, 30, 184-193. [CrossRef]

122. Cermak, N.M.; Res, P.T.; de Groot, L.C.; Saris, W.H.; van Loon, L.J. Protein supplementation augments the adaptive response of skeletal muscle to resistance-type exercise training: A meta-analysis. Am. J. Clin. Nutr. 2012, 96, 1454-1464. [CrossRef] [PubMed]

123. Davies, R.W.; Carson, B.P.; Jakeman, P.M. The Effect of Whey Protein Supplementation on the Temporal Recovery of Muscle Function Following Resistance Training: A Systematic Review and Meta-Analysis. Nutrients 2018, 10, 221. [CrossRef] [PubMed]

124. Finger, D.; Goltz, F.R.; Umpierre, D.; Meyer, E.; Rosa, L.H.; Schneider, C.D. Effects of protein supplementation in older adults undergoing resistance training: A systematic review and meta-analysis. Sports Med. 2015, 45, 245-255. [CrossRef] 
125. Miller, P.E.; Alexander, D.D.; Perez, V. Effects of whey protein and resistance exercise on body composition: A meta-analysis of randomized controlled trials. J. Am. Coll. Nutr. 2014, 33, 163-175. [CrossRef] [PubMed]

126. Naclerio, F.; Larumbe-Zabala, E. Effects of Whey Protein Alone or as Part of a Multi-ingredient Formulation on Strength, Fat-Free Mass, or Lean Body Mass in Resistance-Trained Individuals: A Meta-analysis. Sports Med. 2016, 46, 125-137. [CrossRef] [PubMed]

127. Nissen, S.L.; Sharp, R.L. Effect of dietary supplements on lean mass and strength gains with resistance exercise: A meta-analysis. J. Appl. Physiol. 2003, 94, 651-659. [CrossRef] [PubMed]

128. Schoenfeld, B.J.; Aragon, A.A.; Krieger, J.W. The effect of protein timing on muscle strength and hypertrophy: A meta-analysis. J. Int. Soc. Sports Nutr. 2013, 10, 53. [CrossRef]

129. Thomas, D.K.; Quinn, M.A.; Saunders, D.H.; Greig, C.A. Protein Supplementation Does Not Significantly Augment the Effects of Resistance Exercise Training in Older Adults: A Systematic Review. J. Am. Med. Dir. Assoc. 2016, 17, 959.e1-959.e9. [CrossRef]

130. Schoenfeld, B.J.; Ogborn, D.; Krieger, J.W. Effects of Resistance Training Frequency on Measures of Muscle Hypertrophy: A Systematic Review and Meta-Analysis. Sports Med. 2016, 46, 1689-1697. [CrossRef]

131. Schoenfeld, B.J.; Grgic, J.; Krieger, J. How many times per week should a muscle be trained to maximize muscle hypertrophy? A systematic review and meta-analysis of studies examining the effects of resistance training frequency. J. Sports Sci. 2018, 1-10. [CrossRef]

132. Schoenfeld, B.J.; Contreras, B.; Krieger, J.; Grgic, J.; Delcastillo, K.; Belliard, R.; Alto, A. Resistance Training Volume Enhances Muscle Hypertrophy but Not Strength in Trained Men. Med. Sci. Sports Exerc. 2019, 51, 94-103. [CrossRef] [PubMed]

133. Grgic, J.; Mikulic, P.; Podnar, H.; Pedisic, Z. Effects of linear and daily undulating periodized resistance training programs on measures of muscle hypertrophy: A systematic review and meta-analysis. PeerJ 2017, 5, e3695. [CrossRef] [PubMed]

134. Lexell, J.; Taylor, C.C.; Sjostrom, M. What is the cause of the ageing atrophy? Total number, size and proportion of different fiber types studied in whole vastus lateralis muscle from 15- to 83-year-old men. J. Neurol. Sci. 1988, 84, 275-294. [CrossRef]

135. Akima, H.; Kano, Y.; Enomoto, Y.; Ishizu, M.; Okada, M.; Oishi, Y.; Katsuta, S.; Kuno, S. Muscle function in 164 men and women aged 20-84 yr. Med. Sci. Sports Exerc. 2001, 33, 220-226. [CrossRef] [PubMed]

136. Moore, D.R.; Churchward-Venne, T.A.; Witard, O.; Breen, L.; Burd, N.A.; Tipton, K.D.; Phillips, S.M. Protein ingestion to stimulate myofibrillar protein synthesis requires greater relative protein intakes in healthy older versus younger men. J. Gerontol. Ser. A Biol. Sci. Med Sci. 2015, 70, 57-62. [CrossRef]

137. Ogasawara, R.; Yasuda, T.; Ishii, N.; Abe, T. Comparison of muscle hypertrophy following 6-month of continuous and periodic strength training. Eur. J. Appl. Physiol. 2013, 113, 975-985. [CrossRef]

138. Alway, S.E.; Grumbt, W.H.; Stray-Gundersen, J.; Gonyea, W.J. Effects of resistance training on elbow flexors of highly competitive bodybuilders. J. Appl. Physiol. 1992, 72, 1512-1521. [CrossRef]

139. Bycura, D.; Feito, Y.; Prather, C. Motivational Factors in CrossFit ${ }^{\circledR}$ Training Participation. Health Behav. Policy Rev. 2017, 4, 539-550. [CrossRef]

140. Wikstrom-Frisen, L.; Boraxbekk, C.J.; Henriksson-Larsen, K. Effects on power, strength and lean body mass of menstrual/oral contraceptive cycle based resistance training. J. Sports Med. Phys. Fit. 2017, 57, 43-52.

141. Sung, E.; Han, A.; Hinrichs, T.; Vorgerd, M.; Manchado, C.; Platen, P. Effects of follicular versus luteal phase-based strength training in young women. Springerplus 2014, 3, 668. [CrossRef]

142. Krieger, J.W. Single vs. multiple sets of resistance exercise for muscle hypertrophy: A meta-analysis. J. Strength Cond. Res. 2010, 24, 1150-1159. [CrossRef] [PubMed]

143. Fisher, J. Beware the Meta-Analysis: Is Multiple Set Training Really Better than Single Set Training for Muscle Hypertrophy? J. Exerc. Physiol. Online 2012, 15, 23-30.

144. Burd, N.A.; Holwerda, A.M.; Selby, K.C.; West, D.W.; Staples, A.W.; Cain, N.E.; Cashaback, J.G.; Potvin, J.R.; Baker, S.K.; Phillips, S.M. Resistance exercise volume affects myofibrillar protein synthesis and anabolic signalling molecule phosphorylation in young men. J. Physiol. 2010, 588, 3119-3130. [CrossRef]

145. Dankel, S.J.; Mattocks, K.T.; Jessee, M.B.; Buckner, S.L.; Mouser, J.G.; Counts, B.R.; Laurentino, G.C.; Loenneke, J.P. Frequency: The Overlooked Resistance Training Variable for Inducing Muscle Hypertrophy? Sports Med. 2017, 47, 799-805. [CrossRef] [PubMed] 
146. Abe, T.; Kojima, K.; Kearns, C.F.; Yohena, H.; Fukuda, J. Whole body muscle hypertrophy from resistance training: Distribution and total mass. Br. J. Sports Med. 2003, 37, 543-545. [CrossRef]

147. Cupeiro, C.; Rubio-arias, J.A.; Ramos-campo, D.; Alcaraz, P.E.; Benito, P.J. Comparison of different measurement techniques for quantifying whole body muscle hypertrophy in men. In Book of Abstracts of the 24th Annual Congress of the European College of Sport Science; Bunc, V., Tsolakidis, E., Eds.; ECSS: Prague, Czech Republic, 2019. 